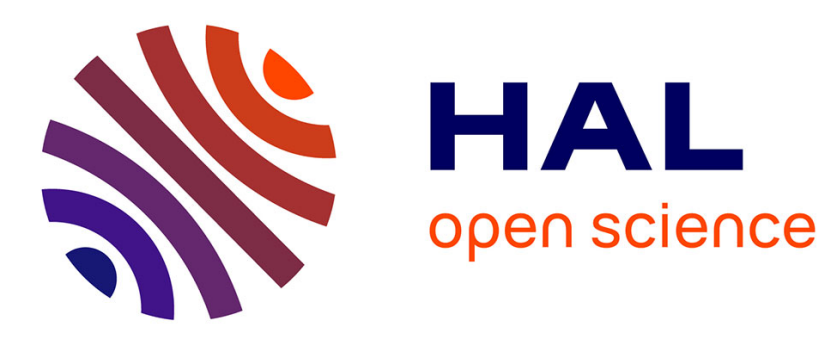

\title{
Nonlinear analysis of periodic waves in a neural field model
}

\author{
S Budzinskiy, A Beuter, Vitaly Volpert
}

\section{To cite this version:}

S Budzinskiy, A Beuter, Vitaly Volpert. Nonlinear analysis of periodic waves in a neural field model.

Chaos: An Interdisciplinary Journal of Nonlinear Science, 2020, 10.1063/5.0012010 . hal-03019102

\section{HAL Id: hal-03019102 \\ https://hal.science/hal-03019102}

Submitted on 23 Nov 2020

HAL is a multi-disciplinary open access archive for the deposit and dissemination of scientific research documents, whether they are published or not. The documents may come from teaching and research institutions in France or abroad, or from public or private research centers.
L'archive ouverte pluridisciplinaire HAL, est destinée au dépôt et à la diffusion de documents scientifiques de niveau recherche, publiés ou non, émanant des établissements d'enseignement et de recherche français ou étrangers, des laboratoires publics ou privés. 


\author{
Nonlinear analysis of periodic waves in a neural field model \\ S. Budzinskiy, 1,2,3 A. Beuter, 4, 5, a) and V. Volpert ${ }^{6,7,3}$ \\ ${ }^{1)}$ Faculty of Computational Mathematics and Cybernetics, Lomonosov Moscow State University, Leninskie Gory 1, \\ 119991 Moscow, Russia \\ ${ }^{2)}$ Marchuk Institute of Numerical Mathematics RAS, Gubkina St. 8, 119333 Moscow, \\ Russia \\ ${ }^{3)}$ Peoples' Friendship University of Russia (RUDN University), Miklukho-Maklaya St. 6, 117198 Moscow, \\ Russia \\ 4) Bordeaux INP, Bordeaux, France \\ ${ }^{5)}$ CorStim SAS, Montpellier, France \\ 6) Institut Camille Jordan, UMR 5208 CNRS, University Lyon 1, 69622 Villeurbanne, France \\ 7) INRIA Team Dracula, INRIA Lyon La Doua, 69603 Villeurbanne, France \\ (Dated: 5 August 2020)
}

Various types of brain activity, including motor, visual, and language, are accompanied by the propagation of periodic waves of electric potential in the cortex, possibly providing the synchronization of the epicenters involved in these activities. One example is cortical electrical activity propagating during sleep and described as traveling waves ${ }^{1}$. These waves modulate cortical excitability as they progress. Clinically-related examples include cortical spreading depression in which a wave of depolarization propagates in migraine but also in stroke, hemorrhage or traumatic brain injury ${ }^{2}$. Here we consider the possible role of epicenters and explore a neural field model with two nonlinear integro-differential equations for the distributions of activating and inhibiting signals. It is studied with symmetric connectivity functions characterizing signal exchange between two populations of neurons, excitatory and inhibitory. Bifurcation analysis is used to investigate the emergence of periodic traveling waves and of standing oscillations from the stationary, spatially homogeneous solutions, and the stability of these solutions. Both types of solutions can be started by local oscillations indicating a possible role of epicenters in the initiation of wave propagation.

The concept of periodic traveling waves appeared in the early 1970's and since that time investigators have actively explored the mechanisms, computational principles and functional role of these waveforms ${ }^{3,4}$. This paper focuses on how modeling information can be used to guide electrical stimulation in the targeted manipulation of brain networks in human patients affected by neurological disorders. We explore a neural field model with two nonlinear integro-differential equations and perform a bifurcation analysis. Results suggest that it is possible to determine the direction of propagation and that epicenters may play a role in the initiation of wave propagating in the cortex. These results will guide the determination of the optimal localization to apply external brain stimulation (forcing term) to restore wave propagation in damaged cortical tissue.

\section{INTRODUCTION}

All types of brain activity are accompanied by formation of spatiotemporal patterns of electric potential in the cerebral cortex, in particular, wave fronts and periodic waves ${ }^{5-7}$. They provide subthreshold depolarization to individual neurons and increase their spiking probability. They possibly synchronize different parts of the brain and organize spatial phase distributions in a population of neurons ${ }^{8}$. Traveling waves can

a)Electronic mail: anne.beuter@wanadoo.fr also be detected from the phase relations between oscillations recorded in different cortical regions 9 .

EEG recordings from the cortical surface in awake neurosurgical patients performing a memory task can show focal regions or clusters of $2-15 \mathrm{~Hz}$ oscillations ${ }^{10}$. These oscillations formed traveling waves that propagated at about $0.25-0.75 \mathrm{~m} / \mathrm{s}$ and correlated with the subject's performance. In other words, they guide the spatial propagation of neural activity demonstrating large scale spatially coordinated oscillations.

The relevance of traveling waves is actively explored in patients with stroke, subarachnoid hemorrhage or traumatic brain injury. The occurrence of spreading depression or depolarization in these pathologies is a well documented phenomenon. Spreading depression or depolarization of neurons and glial cells is also reported in patients with migraine ${ }^{2}$. However, the relevance of traveling waves is also explored in healthy subjects. Indeed traveling waves have been observed in processes involving cognition (such as perception, learning, recognition, memory, language) (see for example Ref. 10 for working memory or Ref. 11 for language) but also in motor behaviors $^{9,12}$ or vision $^{13}$.

For each human subject the examination of slow waves of cortical activity during sleep-awake states using simultaneous scalp EEG and intracranial recordings allowed the identification of the set of intracranial contacts involved in a larger percentage of detected events ${ }^{14}$. These contacts are called «hubs» because the wave had large probability of passing through the region close to the contact. Using probabilities they were able to reconstruct a preferential propagation network for each subject. Slow waves have been reported to propagate across cortical areas at about $1 \mathrm{~m} / \mathrm{s}$ with multiple propagation paths and several points of origin ${ }^{14}$. These waves appear to shape and 
strengthen neuronal networks. There are numerous other evidences of wave propagation during brain activity ${ }^{11,15}$.

There are two main classes of cortical activity which are described by neural mass models and neural field models (see, e.g., Ref. 16). The former is based on a discrete representation of brain networks with spatially homogeneous neural elements connected to each other. In neural field models, cerebral cortex is considered as a continuous medium with a nonlocal interaction due to neuron connectivity. Such models were first introduced in Ref. 17 followed by many others for one or two neuron populations ${ }^{18-20}$, discrete or distributed time delay ${ }^{20,21}$, linear adaptation ${ }^{22}$, refractoriness ${ }^{23}$, and other questions ${ }^{22,24-27}$.

In this work we consider the model

$$
\begin{aligned}
& \frac{\partial u}{\partial t}=P_{1,1} * \psi_{1}(u)-P_{1,2} * \psi_{2}(v)-\sigma u, \\
& \frac{\partial v}{\partial t}=P_{2,1} * \psi_{1}(u)-P_{2,2} * \psi_{2}(v)-\sigma v,
\end{aligned}
$$

where

$$
P_{i, j}(x)=a_{i, j} e^{-b_{i, j}|x|}, i, j=1,2,
$$

$a_{i, j}, b_{i, j}$, and $\sigma$ are positive constants, $\psi_{1}(u)$ and $\psi_{2}(v)$ are bounded smooth growing (sigmoid, i.e. having an S-shaped curve) functions on the whole real axis, $*$ denotes convolution. The variables $u$ and $v$ characterize the activity of the neural population of excitatory and inhibitory neurons, respectively. System (1) represents a particular case without time delay of the model studied in Ref. 28. Considered on the whole real axis, under some natural conditions, it has a stationary, spatially homogeneous solution $\left(u_{0}, v_{0}\right)$. This solution can be stable or unstable depending on the values of parameters. In particular, it can lose its stability due to a simple real eigenvalue or a pair of complex conjugate eigenvalues crossing the imaginary axis. Linear stability analysis of the stationary homogeneous solution was carried out in Ref. 28. Here we are interested in nonlinear bifurcation analysis allowing the determination of stability of the bifurcating solutions. We will obtain the conditions providing the existence and stability of standing and periodic traveling waves.

Furthermore, we are interested in the initiation of the periodic waves by space localized oscillations. From the biological point of view, they correspond to the oscillations in the hubs, which process received information and send it to other hubs in the form of traveling waves. We show that wave initiation occurs depending on the properties of the localized oscillations.

\section{SPECTRUM OF THE LINEARIZED EQUATIONS}

Let $\left(u_{0}(\sigma), v_{0}(\sigma)\right)$ be a pair of constants satisfying

$$
\begin{aligned}
& \sigma u_{0}=2 \frac{a_{1,1}}{b_{1,1}} \psi_{1}\left(u_{0}\right)-2 \frac{a_{1,2}}{b_{1,2}} \psi_{2}\left(v_{0}\right), \\
& \sigma v_{0}=2 \frac{a_{2,1}}{b_{2,1}} \psi_{1}\left(u_{0}\right)-2 \frac{a_{2,2}}{b_{2,2}} \psi_{2}\left(v_{0}\right) .
\end{aligned}
$$

Then $\left(u_{0}(\sigma), v_{0}(\sigma)\right)$ are solutions to (1). By shifting the functions

$$
u(x, t)=u_{0}(\sigma)+\tilde{u}(x, t), \quad v(x, t)=v_{0}(\sigma)+\tilde{v}(x, t),
$$

linearizing the equations (1), and dropping the tildes, we get

$$
\begin{aligned}
& \frac{\partial u}{\partial t}=\psi_{1}^{\prime}\left(u_{0}(\sigma)\right) P_{1,1} * u-\psi_{2}^{\prime}\left(v_{0}(\sigma)\right) P_{1,2} * v-\sigma u(x, t), \\
& \frac{\partial v}{\partial t}=\psi_{1}^{\prime}\left(u_{0}(\sigma)\right) P_{2,1} * u-\psi_{2}^{\prime}\left(v_{0}(\sigma)\right) P_{2,2} * v-\sigma v(x, t) .
\end{aligned}
$$

Looking for solutions in the form of

$$
u(x, t)=\exp (\lambda t) u(x), \quad v(x, t)=\exp (\lambda t) v(x)
$$

and after applying the Fourier transform, we obtain the characteristic equations

$\psi_{1}^{\prime}\left(u_{0}(\sigma)\right) \hat{P}_{1,1}(\xi) \hat{u}(\xi)-\psi_{2}^{\prime}\left(v_{0}(\sigma)\right) \hat{P}_{1,2}(\xi) \hat{v}(\xi)=(\sigma+\lambda) \hat{u}(\xi)$, $\psi_{1}^{\prime}\left(u_{0}(\sigma)\right) \hat{P}_{2,1}(\xi) \hat{u}(\xi)-\psi_{2}^{\prime}\left(v_{0}(\sigma)\right) \hat{P}_{2,2}(\xi) \hat{v}(\xi)=(\sigma+\lambda) \hat{v}(\xi)$, where

$$
\hat{P}_{i, j}(\xi)=\frac{2 a_{i, j} b_{i, j}}{b_{i, j}^{2}+\xi^{2}} .
$$

Let $\mu=\mu(\xi ; \sigma)=\sigma+\lambda(\xi ; \sigma) \in \mathbb{C}$ and

$$
\begin{aligned}
& \hat{Q}_{i, 1}(\xi ; \sigma)=\psi_{1}^{\prime}\left(u_{0}(\sigma)\right) \hat{P}_{i, 1}(\xi), \\
& \hat{Q}_{i, 2}(\xi ; \sigma)=\psi_{2}^{\prime}\left(v_{0}(\sigma)\right) \hat{P}_{i, 2}(\xi) .
\end{aligned}
$$

Then the characteristic polynomial becomes

$$
\begin{aligned}
\mu^{2}-\left(\hat{Q}_{1,1}(\xi)\right. & \left.-\hat{Q}_{2,2}(\xi)\right) \mu- \\
& -\left(\hat{Q}_{1,1}(\xi) \hat{Q}_{2,2}(\xi)-\hat{Q}_{1,2}(\xi) \hat{Q}_{2,1}(\xi)\right)=0
\end{aligned}
$$

with two roots

$$
\begin{aligned}
\mu(\xi)= & \frac{1}{2}\left(\hat{Q}_{1,1}(\xi)-\hat{Q}_{2,2}(\xi)\right) \pm \\
& \pm \frac{1}{2} \sqrt{\left(\hat{Q}_{1,1}(\xi)+\hat{Q}_{2,2}(\xi)\right)^{2}-4 \hat{Q}_{1,2}(\xi) \hat{Q}_{2,1}(\xi)} .
\end{aligned}
$$

If the cross-connectivity functions $P_{1,2}(x)$ and $P_{2,1}(x)$ are strong enough (their amplitudes $a_{1,2}$ and $a_{2,1}$ are large enough), we can ensure that the expression under the square root is always negative

$$
\left(\hat{Q}_{1,1}(\xi)+\hat{Q}_{2,2}(\xi)\right)^{2}-4 \hat{Q}_{1,2}(\xi) \hat{Q}_{2,1}(\xi)<0, \quad-\infty<\xi<\infty,
$$

which leads to complex values of $\mu(\xi)$ such that

$$
\begin{aligned}
\operatorname{Re} \mu(\xi) & =\frac{1}{2}\left(\hat{Q}_{1,1}(\xi)-\hat{Q}_{2,2}(\xi)\right) \\
\operatorname{Im} \mu(\xi) & = \pm \frac{1}{2} \sqrt{4 \hat{Q}_{1,2}(\xi) \hat{Q}_{2,1}(\xi)-\left(\hat{Q}_{1,1}(\xi)+\hat{Q}_{2,2}(\xi)\right)^{2}} .
\end{aligned}
$$

Consider a function $f(\xi): \mathbb{R} \rightarrow \mathbb{R}$ that is related to the real part of the characteristic values $\mu(\xi)$ :

$$
f(\xi)=\frac{\alpha_{1}}{\beta_{1}+\xi^{2}}-\frac{\alpha_{2}}{\beta_{2}+\xi^{2}}, \quad \alpha_{i}>0, \quad \beta_{i}>0 .
$$

We are interested in its extreme values and the corresponding frequency $\xi$ where they are attained. 
Lemma 1. If $\alpha_{1}=\alpha_{2}$ or $\beta_{1}=\beta_{2}$ then $|f(\xi)| \leq|f(0)|$ for all $\xi$.

Proof. Let $\alpha_{1}=\alpha_{2}=\alpha$. Then

$$
f(\xi)=\frac{\alpha\left(\beta_{2}-\beta_{1}\right)}{\left(\beta_{1}+\xi^{2}\right)\left(\beta_{2}+\xi^{2}\right)} .
$$

Unless $\beta_{1}=\beta_{2}$ and $f(\xi) \equiv 0,|f(\xi)|$ is monotonically decreasing on $\xi>0$ and increasing on $\xi<0$.

Now let $\beta_{1}=\beta_{2}=\beta$. Then

$$
f(\xi)=\frac{\alpha_{1}-\alpha_{2}}{\beta+\xi^{2}} .
$$

Unless $\alpha_{1}=\alpha_{2}$ and $f(\xi) \equiv 0,|f(\xi)|$ is monotonically decreasing on $\xi>0$ and increasing on $\xi<0$.

Let $\alpha_{1} \neq \alpha_{2}$ and $\beta_{1} \neq \beta_{2}$; we introduce

$$
\beta_{3}=\frac{\alpha_{1} \beta_{2}-\alpha_{2} \beta_{1}}{\alpha_{1}-\alpha_{2}}
$$

and note that it can take any real value, not necessarily positive ones. Then the derivative of $f(\xi)$ is

$$
\begin{aligned}
f^{\prime}(\xi) & =2 \xi\left(\alpha_{1}-\alpha_{2}\right) \times \\
& \times \frac{\left(\beta_{1}+\xi^{2}\right)\left(\beta_{2}+\xi^{2}\right)-\left(\beta_{3}+\xi^{2}\right)\left(\beta_{1}+\beta_{2}+2 \xi^{2}\right)}{\left(\beta_{1}+\xi^{2}\right)^{2}\left(\beta_{2}+\xi^{2}\right)^{2}} .
\end{aligned}
$$

It vanishes at $\xi=0$ and, possibly, at

$$
\xi^{2}=-\beta_{3} \pm \sqrt{\left(\beta_{3}-\beta_{1}\right)\left(\beta_{3}-\beta_{2}\right)} \equiv \eta_{ \pm}
$$

if the right hand side is real and positive.

Lemma 2. Both $\eta_{+}$and $\eta_{-}$are real whenever

$$
\beta_{3} \leq \min \left(\beta_{1}, \beta_{2}\right) \text { or } \beta_{3} \geq \max \left(\beta_{1}, \beta_{2}\right) .
$$

The value $\eta_{-}$is negative when it is real, i.e. whenever

$$
\beta_{3} \leq \min \left(\beta_{1}, \beta_{2}\right) \text { or } \beta_{3} \geq \max \left(\beta_{1}, \beta_{2}\right) .
$$

The value $\eta_{+}$is nonpositive whenever

$$
\frac{\beta_{1} \beta_{2}}{\beta_{1}+\beta_{2}} \leq \beta_{3} \leq \min \left(\beta_{1}, \beta_{2}\right) \text { or } \beta_{3} \geq \max \left(\beta_{1}, \beta_{2}\right) .
$$

Proof. The first statement is obvious. Let us assume that $\eta_{+}, \eta_{-} \in \mathbb{R}$. For the second statement,

1. if $\beta_{3} \geq 0$ then $\eta_{-}<0$ if and only if

$$
\beta_{3}>-\sqrt{\left(\beta_{3}-\beta_{1}\right)\left(\beta_{3}-\beta_{2}\right)},
$$

so $0 \leq \beta_{3} \leq \min \left(\beta_{1}, \beta_{2}\right)$ or $\beta_{3} \geq \max \left(\beta_{1}, \beta_{2}\right)$;

2. if $\beta_{3}<0$ then $\eta_{-}<0$ if and only if

$$
\beta_{1} \beta_{2}-\beta_{3}\left(\beta_{1}+\beta_{2}\right)>0,
$$

so simply $\beta_{3}<0$.
For the third statement, $\eta_{+} \leq 0$ if and only if

$$
\beta_{1} \beta_{2}-\beta_{3}\left(\beta_{1}+\beta_{2}\right) \leq 0
$$

whence the result follows.

Lemma 3. Let $\alpha_{1} \neq \alpha_{2}, \beta_{1} \neq \beta_{2}$, and $\beta_{3}<\beta_{1} \beta_{2} /\left(\beta_{1}+\beta_{2}\right)$. The derivative $f^{\prime}(\xi)$ vanishes at only 3 points:

$$
\xi=0 \text { and } \xi= \pm \sqrt{-\beta_{3}+\sqrt{\left(\beta_{3}-\beta_{1}\right)\left(\beta_{3}-\beta_{2}\right)}} .
$$

Proof. Since $\alpha_{1} \neq \alpha_{2}$ and $\beta_{1} \neq \beta_{2}, f^{\prime}(\xi)$ turns to zero if and only if $\xi=0$ or $\xi^{2}=\eta_{ \pm}$. Lemma 2 states that $\eta_{-}<0$ and $\eta_{+}>0$ when $\beta_{3}<\beta_{1} \beta_{2} /\left(\beta_{1}+\beta_{2}\right)$. So $f^{\prime}(\xi)=0$ at $\xi=0$ and $\xi= \pm \sqrt{\eta_{+}}$.

Since

$$
f^{\prime}(\xi)=2\left(\alpha_{1}-\alpha_{2}\right) \frac{\beta_{1} \beta_{2}-\beta_{3}\left(\beta_{1}+\beta_{2}\right)}{\beta_{1}^{2} \beta_{2}^{2}} \xi+O\left(\xi^{2}\right)
$$

in the vicinity of the origin, the function $f(\xi)$ has global maxima at

$$
\xi^{2}=-\beta_{3}+\sqrt{\left(\beta_{3}-\beta_{1}\right)\left(\beta_{3}-\beta_{2}\right)}
$$

when $\alpha_{1}>\alpha_{2}$, and global minima when $\alpha_{1}<\alpha_{2}$.

Let

$$
\alpha_{1}=\psi_{1}^{\prime}\left(u_{0}(\sigma)\right) a_{1,1} b_{1,1}, \quad \beta_{1}=b_{1,1}^{2}
$$

and

$$
\alpha_{2}=\psi_{2}^{\prime}\left(v_{0}(\sigma)\right) a_{2,2} b_{2,2}, \quad \beta_{2}=b_{2,2}^{2},
$$

then $\operatorname{Re} \mu(\xi)=f(\xi)$ and the largest real part $\sup _{\xi} \operatorname{Re} \mu(\xi)$ is attained at $\pm \xi_{*}(\sigma)$ :

$$
\begin{aligned}
& \xi_{*}(\sigma)=\sqrt{-\beta_{3}(\sigma)+\sqrt{\left(\beta_{3}(\sigma)-b_{1,1}^{2}\right)\left(\beta_{3}(\sigma)-b_{2,2}^{2}\right)}}, \\
& \beta_{3}(\sigma)=\frac{\psi_{1}^{\prime}\left(u_{0}(\sigma)\right) a_{1,1} b_{1,1} b_{2,2}^{2}-\psi_{2}^{\prime}\left(v_{0}(\sigma)\right) a_{2,2} b_{2,2} b_{1,1}^{2}}{\psi_{1}^{\prime}\left(u_{0}(\sigma)\right) a_{1,1} b_{1,1}-\psi_{2}^{\prime}\left(v_{0}(\sigma)\right) a_{2,2} b_{2,2}} .
\end{aligned}
$$

Thus $\sup _{\xi} \operatorname{Re} \lambda(\xi ; \sigma)=\operatorname{Re} \lambda\left(\xi_{*}(\sigma)\right)=f\left(\xi_{*}(\sigma)\right)-\sigma$.

Theorem 1. Let $a_{12}, a_{21} \gg 1$ and

$$
\frac{b_{22}}{b_{11}}<\frac{\psi_{1}^{\prime}\left(u_{0}(\sigma)\right) a_{11}}{\psi_{2}^{\prime}\left(v_{0}(\sigma)\right) a_{22}}<\left(\frac{b_{11}}{b_{22}}\right)^{3} .
$$

Then the spectrum is complex $\operatorname{Im} \lambda(\xi ; \sigma) \neq 0$ and $\operatorname{Re} \lambda(\xi ; \sigma)<\operatorname{Re} \lambda\left(\xi_{*}(\sigma) ; \sigma\right)$ for all $\xi \neq \pm \xi_{*}(\sigma)$.

Proof. Follows from Lemmas 1-3 once we rewrite the constraint $\beta_{3}<\beta_{1} \beta_{2} /\left(\beta_{1}+\beta_{2}\right)$. 


\section{HOPF BIFURCATION AND NORMAL FORM ANALYSIS}

Fix a value $\sigma_{*}>0$ and let $\sigma \in \mathbb{R}$ be its small perturbation. With a slight abuse of notation we will write $f(\sigma)$ for functions of $\sigma$ that we would previously call $f\left(\sigma_{*}+\sigma\right)$. Assume that

$$
\operatorname{Re} \lambda(\xi ; 0)<\operatorname{Re} \lambda\left(\xi_{*}(0) ; 0\right), \quad \xi \neq \xi_{*}(0),
$$

and

$$
\lambda\left(\xi_{*}(0) ; 0\right)= \pm \mathrm{i} \omega_{*} \neq 0 .
$$

Condition (H1) follows from Theorem 1 while the Hopf bifurcation condition (H2) can be satisfied with a careful choice of parameters. We also assume the transversality condition

$$
\begin{aligned}
\left(\frac{d}{d \sigma} \operatorname{Re} \lambda\left(\xi_{*}(0) ; \sigma\right)\right) & = \\
=-1 & +\frac{1}{2} \psi_{1}^{\prime \prime}\left(u_{0}(0)\right) u_{0}^{\prime}(0) \hat{P}_{11}\left(\xi_{*}(0)\right)- \\
& \quad-\frac{1}{2} \psi_{2}^{\prime \prime}\left(v_{0}(0)\right) v_{0}^{\prime}(0) \hat{P}_{22}\left(\xi_{*}(0)\right) \neq 0 .
\end{aligned}
$$

That is the pair of imaginary eigenvalues passes the imaginary axis with nonzero speed.

Let $\xi_{*}=\xi_{*}(0)$ and $l_{*}=2 \pi / \xi_{*}$. Consider the Lebesgue space $L^{2}\left(0, l_{*}\right)$ with standard inner product and norm:

$$
\langle f, g\rangle_{L^{2}\left(0, l_{*}\right)}=\int_{0}^{l_{*}} f \bar{g} d x, \quad\|f\|_{L^{2}\left(0, l_{*}\right)}=\sqrt{\langle f, f\rangle_{L^{2}\left(0, l_{*}\right)}} .
$$

The complex exponentials $e_{n}=\frac{1}{\sqrt{l_{*}}} e^{\mathrm{i} n \xi_{*} x}$ form an orthonormal basis in $L^{2}\left(0, L_{*}\right)$. Also, consider the Sobolev space of $l_{*^{-}}$ periodic twice-differentiable functions $H_{p e r}^{2}\left(0, l_{*}\right)$ with inner product

$$
\langle f, g\rangle_{H_{p e r}^{2}\left(0, l_{*}\right)}=\sum_{n \in \mathbb{Z}}\left(1+n^{4} \xi_{*}^{4}\right)\left\langle f, e_{n}\right\rangle_{L^{2}\left(0, l_{*}\right)}\left\langle e_{n}, g\right\rangle_{L^{2}\left(0, l_{*}\right)}
$$

and Euclidean norm. We consider smooth functions to make the analysis extendable to diffusive models.

Let

$$
w=\left[\begin{array}{l}
u \\
v
\end{array}\right] \in X=\left(H_{p e r}^{2}\left(0, l_{*}\right)\right)^{2}
$$

with inner product

$$
\langle w, \tilde{w}\rangle_{X}=\left\langle w_{1}, \tilde{w}_{1}\right\rangle_{H_{p e r}^{2}\left(0, l_{*}\right)}+\left\langle w_{2}, \tilde{w}_{2}\right\rangle_{H_{p e r}^{2}\left(0, l_{*}\right)}
$$

where $w_{j}$ and $\tilde{w}_{j}$ are the components of two-dimensional vector functions.

Let $M_{k}(\sigma) \in \mathbb{R}^{2 \times 2}$ be diagonal matrices

$$
M_{k}(\sigma)=\frac{1}{k !}\left[\begin{array}{cc}
\psi_{1}^{(k)}\left(u_{0}(\sigma)\right) & 0 \\
0 & -\psi_{2}^{(k)}\left(v_{0}(\sigma)\right)
\end{array}\right]
$$

and $\mathscr{P}_{n} \in \mathbb{R}^{2 \times 2}$ be Fourier filtering matrices

$$
\mathscr{P}_{n}=\left[\begin{array}{ll}
\hat{P}_{11}\left(n \xi_{*}\right) & \hat{P}_{12}\left(n \xi_{*}\right) \\
\hat{P}_{21}\left(n \xi_{*}\right) & \hat{P}_{22}\left(n \xi_{*}\right)
\end{array}\right]
$$

Any function $w \in X \subset\left(L^{2}\left(0, l_{*}\right)\right)^{2}$ can be represented by its Fourier series

$$
w=\sum_{n \in \mathbb{Z}} w_{n} e_{n}, \quad w_{n}=\left[\begin{array}{l}
\left\langle w_{1}, e_{n}\right\rangle_{L^{2}\left(0, l_{*}\right)} \\
\left\langle w_{2}, e_{n}\right\rangle_{L^{2}\left(0, l_{*}\right)}
\end{array}\right] \in \mathbb{C}^{2} .
$$

We can rewrite the initial equations as

$$
w_{t}=L(\sigma) w+F(w, \sigma), \quad w \in X,
$$

where

$$
L(\sigma) w=\sum_{n \in \mathbb{Z}}\left(\mathscr{P}_{n} M_{1}(\sigma)-\sigma_{*}-\sigma\right) w_{n} e_{n}
$$

and

$$
F(w, \sigma)=\sum_{k \geq 2} \sum_{n \in \mathbb{Z}} \mathscr{P}_{n} M_{k}(\sigma)\left(w^{\odot k}\right)_{n} e_{n}
$$

We use $\odot$ to denote the Hadamard (element-wise) product of vectors and write $w^{\odot 2}=w \odot w, w^{\odot 3}=w \odot w \odot w$, etc., for Hadamard products of $w$ with itself.

The characteristic equation at $\sigma=0$ decouples into

$$
\left(\mathscr{P}_{n} M_{1}(0)-\sigma_{*}-\lambda\right) w_{n}=0, \quad n \in \mathbb{Z},
$$

and $\lambda \in \mathbb{C}$ is a characteristic value if there exist $n \in \mathbb{Z}$ and $w_{n} \neq 0 \in \mathbb{C}^{2}$ that satisfy the equation above. Owing to (H2), there is a unique pair of imaginary characteristic values $\pm \mathrm{i} \omega_{*}$ corresponding to $n= \pm 1$. They are degenerate because the connectivity functions are symmetric and the system as a whole is $O(2)$-equivariant. Let $\zeta_{1} \neq 0 \in \mathbb{C}^{2}$ be an eigenvector:

$$
\begin{aligned}
& \left(\mathscr{P}_{1} M_{1}(0)-\sigma_{*}-\mathrm{i} \omega_{*}\right) \zeta_{1}=0, \\
& \zeta_{1}=\left[\begin{array}{ll}
\psi_{2}^{\prime}\left(v_{0}(0)\right) \hat{P}_{12}\left(\xi_{*}\right) & \psi_{1}^{\prime}\left(u_{0}(0)\right) \hat{P}_{11}\left(\xi_{*}\right)-\sigma_{*}-\mathrm{i} \omega_{*}
\end{array}\right]^{T} .
\end{aligned}
$$

Consider vector functions

$$
q_{1}=\zeta_{1} e_{1}, \quad q_{2}=\zeta_{1} e_{-1}, \quad q_{3}=\overline{\zeta_{1}} e_{1}, \quad q_{4}=\overline{\zeta_{1}} e_{-1} .
$$

In pairs, they span the eigenspaces

$$
\begin{aligned}
& \mathscr{N}\left(L(0)-\mathrm{i} \omega_{*}\right)=\operatorname{span}\left\{q_{1}, q_{2}\right\} \subset X, \\
& \mathscr{N}\left(L(0)+\mathrm{i} \omega_{*}\right)=\operatorname{span}\left\{q_{3}, q_{4}\right\} \subset X ;
\end{aligned}
$$

together they span the center subspace

$$
\mathscr{Q}=\operatorname{span}\left\{q_{1}, q_{2}, q_{3}, q_{4}\right\} \subset X .
$$

The basis $\left\{q_{j}\right\}$ is not orthogonal so we will use a biorthogonal system to get a projection operator onto $\mathscr{Q}$. Let $\zeta_{1}^{*} \neq 0 \in \mathbb{C}^{2}$ be an eigenvector of the adjoint matrix

$$
\left(\mathscr{P}_{1} M_{1}(0)-\sigma_{*}-\mathrm{i} \omega_{*}\right)^{H} \zeta_{1}^{*}=0
$$


normalized so that $\left\langle\zeta_{1}, \zeta_{1}^{*}\right\rangle=1$. Further, let

$$
\begin{array}{ll}
q_{1}^{*}=\frac{1}{1+\xi_{*}^{4}} \zeta_{1}^{*} e_{1}, & q_{2}^{*}=\frac{1}{1+\xi_{*}^{4}} \zeta_{1}^{*} e_{-1}, \\
q_{3}^{*}=\frac{1}{1+\xi_{*}^{4}} \overline{\zeta_{1}^{*}} e_{1}, & q_{4}^{*}=\frac{1}{1+\xi_{*}^{4}} \overline{\zeta_{1}^{*}} e_{-1}
\end{array}
$$

be the basis vectors of the eigenspaces

$$
\begin{aligned}
& \mathscr{N}\left(L(0)^{*}+\mathrm{i} \omega_{*}\right)=\operatorname{span}\left\{q_{1}^{*}, q_{2}^{*}\right\} \subset X, \\
& \mathscr{N}\left(L(0)^{*}-\mathrm{i} \omega_{*}\right)=\operatorname{span}\left\{q_{3}^{*}, q_{4}^{*}\right\} \subset X .
\end{aligned}
$$

It is easy to show that $\left\{q_{j}^{*}\right\}$ and $\left\{q_{j}\right\}$ are biorthogonal systems

$$
\left\langle q_{i}, q_{j}^{*}\right\rangle_{X}= \begin{cases}1, & i=j, \\ 0, & i \neq j .\end{cases}
$$

We can thus define a projection operator

$$
\pi: X \rightarrow \mathscr{Q}, \quad \pi w=\sum_{j=1}^{4}\left\langle w, q_{j}^{*}\right\rangle_{X} q_{j}
$$

and a complementary subspace

$$
\tilde{\mathscr{Q}}=\{w \in X: \pi w=0\} .
$$

Then $X=\mathscr{Q} \oplus \tilde{\mathscr{Q}}$.

For a given $w \in X$, let $z \in \mathbb{C}^{4}$ (note that $z_{4}=\overline{z_{1}}$ and $z_{2}=\overline{z_{3}}$ as the functions are real-valued) be the vector of coefficients of $\pi w$ with respect to the basis $\left\{q_{j}\right\}$. Let $\gamma$ be the velocity of the critical characteristic value:

$$
\gamma=\left.\left(\frac{d}{d \sigma} \lambda\left(\xi_{*} ; \sigma\right)\right)\right|_{\sigma=0} .
$$

According to the center manifold theorem, the equations for $z_{1}$ and $z_{3}$ on the manifold have the following normal form ${ }^{29}$ :

$$
\begin{aligned}
& \dot{z}_{1}=\left(\mathrm{i} \omega_{*}+\gamma \sigma\right) z_{1}+c_{1} z_{1}\left|z_{1}\right|^{2}+c_{2} z_{1}\left|z_{3}\right|^{2}+\text { h.o.t. } \\
& \dot{z}_{3}=\left(\mathrm{i} \omega_{*}+\gamma \sigma\right) z_{3}+c_{1} z_{3}\left|z_{3}\right|^{2}+c_{2} z_{3}\left|z_{1}\right|^{2}+\text { h.o.t. }
\end{aligned}
$$

where h.o.t. $=\mathscr{O}\left(\sigma\left(\left|z_{1}\right|^{3}+\left|z_{3}\right|^{3}\right)+\sigma^{2}\left(\left|z_{1}\right|+\left|z_{3}\right|\right)+\left(\left|z_{1}\right|+\right.\right.$ $\left.\left.\left|z_{2}\right|\right)^{5}\right)$. See Appendix A for the computation of the cubic coefficients $c_{1}$ and $c_{2}$ of the normal form.

The relations between $c_{1}$ and $c_{2}$ determine the stability of periodic traveling wave and standing wave solutions ${ }^{29}$. To see this, we make a polar change of coordinates,

$$
z_{1}=\rho_{1} e^{i \theta_{1}}, \quad z_{3}=\rho_{3} e^{i \theta_{3}},
$$

and keep two equations out of four:

$$
\begin{aligned}
& \dot{\rho}_{1}=\rho_{1}\left(\sigma \operatorname{Re} \gamma+\operatorname{Re} c_{1} \rho_{1}^{2}+\operatorname{Re} c_{2} \rho_{3}^{2}\right)+\text { h.o.t. } \\
& \dot{\rho}_{3}=\rho_{3}\left(\sigma \operatorname{Re} \gamma+\operatorname{Re} c_{1} \rho_{3}^{2}+\operatorname{Re} c_{2} \rho_{1}^{2}\right)+\text { h.o.t. }
\end{aligned}
$$

These are the amplitude equations as $\rho_{1}$ and $\rho_{3}$ represent the amplitudes of counterpropagating periodic traveling waves. The system (2) can have up to four equilibria (see Table I),
TABLE I: Equilibria of the amplitude equations (2).

\begin{tabular}{lll}
\hline \hline Type of solution & $\rho_{1}$ & $\rho_{3}$ \\
\hline Constant & 0 & 0 \\
Periodic traveling wave & $\sqrt{-\frac{\sigma \operatorname{Re} \gamma}{\operatorname{Re} c_{1}}}$ & 0 \\
Periodic traveling wave & 0 & $\sqrt{-\frac{\sigma \operatorname{Re} \gamma}{\operatorname{Re} c_{1}}}$ \\
Standing wave & $\sqrt{-\frac{\sigma \operatorname{Re} \gamma}{\operatorname{Rec} c_{1}+\operatorname{Rec} c_{2}}}$ & $\sqrt{-\frac{\sigma \operatorname{Re} \gamma}{\operatorname{Re} c_{1}+\operatorname{Rec} c_{2}}}$ \\
\hline \hline
\end{tabular}

and the stability of these equilibria defines the stability of periodic traveling waves and standing waves.

Let $\sigma \operatorname{Re} \gamma>0$ then

$$
\operatorname{Re} c_{1}<0, \quad \operatorname{Re}\left(c_{1}+c_{2}\right)<0, \quad \operatorname{Re}\left(c_{1}-c_{2}\right)<0
$$

leads to asymptotically stable standing waves while

$$
\operatorname{Re} c_{1}<0, \quad \operatorname{Re}\left(c_{1}+c_{2}\right)<0, \quad \operatorname{Re}\left(c_{1}-c_{2}\right)>0
$$

produces asymptotically stable periodic traveling waves.

\section{ONE CLASS OF PARAMETERS}

The net result of Sections II-III are the explicit expressions of the normal form coefficients, which can be easily evaluated for a given set of parameters. However, these expressions are too cumbersome to aid in the search of suitable parameters. Theorem 1 is also of merely theoretical interest: the condition $a_{1,2}, a_{2,1} \gg 1$ which ensures that the whole spectrum lies in $\mathbb{C} \backslash \mathbb{R}$ is too vague to be verifiable. In this section, we will present and semi-rigorously analyze a particular class of parameters that can be studied in more details.

Let $\psi_{1}=\psi_{2}=\psi$ be a sigmoid function. In our analysis, we will use a number of its properties such as

$$
\psi^{\prime}(0)>0, \quad \psi^{\prime \prime}(0)=0, \quad \psi^{\prime \prime \prime}(0)<0, \quad \psi^{\prime \prime \prime \prime}(0)=0 .
$$

Let $R>0$ be the ratio of parameters

$$
\frac{a_{1,2}}{b_{1,2}}=\frac{a_{2,1}}{b_{2,1}}=\frac{a_{2,2}}{b_{2,2}}=R
$$

and suppose that the ratio $a_{1,1} / b_{1,1}$ is perturbed slightly

$$
\frac{a_{1,1}}{b_{1,1}}=R+\varepsilon, \quad \varepsilon \in \mathbb{R} .
$$

Next, assume that the parameters $b_{i, j}$ are equal

$$
b_{1,1}=b_{1,2}=b_{2,1}=b, \quad b>0,
$$

except for $b_{2,2}$ that is smaller:

$$
b_{2,2}=v b, \quad 0<v<1 .
$$

Lemma 4. For all small values of $\varepsilon$, system (1) has constant solutions

$$
\begin{aligned}
& u_{0}=2 \frac{\psi(0)}{\sigma_{*}}\left(1+2 R \frac{\psi^{\prime}(0)}{\sigma_{*}}\right) \varepsilon+\mathscr{O}\left(\varepsilon^{2}\right), \\
& v_{0}=4 R \frac{\psi(0)}{\sigma_{*}} \frac{\psi^{\prime}(0)}{\sigma_{*}} \varepsilon+\mathscr{O}\left(\varepsilon^{2}\right) .
\end{aligned}
$$


Proof. At $\varepsilon=0$, we have

$$
\sigma_{*} u_{0}=\sigma_{*} v_{0}=2 R\left(\psi\left(u_{0}\right)-\psi\left(v_{0}\right)\right)
$$

and $u_{0}=v_{0}=0$. By the inverse function theorem there is a smooth branch of solutions $\left(u_{0}(\varepsilon), v_{0}(\varepsilon)\right)$ whose first derivatives satisfy the equation

$$
\left[\begin{array}{cc}
\sigma_{*}-2 R \psi^{\prime}(0) & 2 R \psi^{\prime}(0) \\
-2 R \psi^{\prime}(0) & \sigma_{*}+2 R \psi^{\prime}(0)
\end{array}\right]\left[\begin{array}{c}
\frac{d u_{0}}{d \varepsilon}(0) \\
\frac{d v_{0}}{d \varepsilon}(0)
\end{array}\right]=\left[\begin{array}{c}
2 \psi(0) \\
0
\end{array}\right] .
$$

Note that the Jacobian matrix is full-rank for all admissible values of $R, \sigma_{*}$, and $\psi^{\prime}(0)$, being a sum of a scalar matrix and a rank-1 matrix. Its inverse can be computed, for example, with the Sherman-Morrison formula.

\section{A. Spectrum}

By the properties of sigmoid functions,

$$
\psi^{\prime}\left(u_{0}\right)=\psi^{\prime}(0)+\mathscr{O}\left(\varepsilon^{2}\right) . \quad \psi^{\prime}\left(v_{0}\right)=\psi^{\prime}(0)+\mathscr{O}\left(\varepsilon^{2}\right) .
$$

Then

$$
\begin{aligned}
& \hat{Q}_{1,1}(\xi)=2 \psi^{\prime}(0)(R+\varepsilon) \frac{b^{2}}{b^{2}+\xi^{2}}+\mathscr{O}\left(\varepsilon^{2}\right) \\
& \hat{Q}_{1,2}(\xi)=\hat{Q}_{2,1}(\xi)=2 \psi^{\prime}(0) R \frac{b^{2}}{b^{2}+\xi^{2}}+\mathscr{O}\left(\varepsilon^{2}\right), \\
& \hat{Q}_{2,2}(\xi)=2 \psi^{\prime}(0) R \frac{v^{2} b^{2}}{v^{2} b^{2}+\xi^{2}}+\mathscr{O}\left(\varepsilon^{2}\right) .
\end{aligned}
$$

Recall that the characteristic values are

$$
\begin{aligned}
\mu(\xi)= & \frac{1}{2}\left(\hat{Q}_{1,1}(\xi)-\hat{Q}_{2,2}(\xi)\right) \pm \\
& \pm \frac{1}{2} \sqrt{\left(\hat{Q}_{1,1}(\xi)+\hat{Q}_{2,2}(\xi)\right)^{2}-4 \hat{Q}_{1,2}(\xi) \hat{Q}_{2,1}(\xi)}
\end{aligned}
$$

Consider the expression under the square root

$$
\begin{aligned}
& \left(\hat{Q}_{1,1}(\xi)+\hat{Q}_{2,2}(\xi)\right)^{2}-4 \hat{Q}_{1,2}(\xi) \hat{Q}_{2,1}(\xi)= \\
& =\left(\frac{2 \psi^{\prime}(0) R b^{2}}{\left(b^{2}+\xi^{2}\right)}\right)^{2}\left[\left(1+v^{2} \frac{b^{2}+\xi^{2}}{v^{2} b^{2}+\xi^{2}}\right)^{2}+\right. \\
& \left.\quad+2\left(1+v^{2} \frac{b^{2}+\xi^{2}}{v^{2} b^{2}+\xi^{2}}\right) \varepsilon-4 R^{2}\right]+\mathscr{O}\left(\varepsilon^{2}\right) .
\end{aligned}
$$

It has the same sign as

$$
\begin{aligned}
\left(1+v^{2} \frac{b^{2}+\xi^{2}}{v^{2} b^{2}+\xi^{2}}\right) & -\left(2-\frac{\varepsilon}{R}+\mathscr{O}\left(\varepsilon^{2}\right)\right)= \\
& =\frac{\varepsilon}{R}-\left(1-v^{2}\right) \frac{\xi^{2}}{v^{2} b^{2}+\xi^{2}}+\mathscr{O}\left(\varepsilon^{2}\right) .
\end{aligned}
$$

Since $\frac{\xi^{2}}{v^{2} b^{2}+\xi^{2}}$ is monotonically increasing as a function of $\xi^{2}$, there is a value

$$
\xi_{0}^{2}=\frac{v^{2} b^{2}}{1-v^{2}} \frac{\varepsilon}{R}+\mathscr{O}\left(\varepsilon^{2}\right)
$$

if $0<\varepsilon / R<1-v^{2}$, such that the expression under the square root is positive for $0 \leq \xi^{2}<\xi_{0}^{2}$ and negative for $\xi^{2}>\xi_{0}^{2}$. It follows that

$$
\mu(\xi) \in\left\{\begin{array}{l}
\mathbb{R}, \quad 0 \leq \xi^{2}<\xi_{0}^{2}, \\
\mathbb{C} \backslash \mathbb{R}, \quad \xi^{2}>\xi_{0}^{2} .
\end{array}\right.
$$

If $\varepsilon<0$, the whole spectrum lies in $\mathbb{C} \backslash \mathbb{R}$ and we can apply Theorem 1 to show that

$$
\operatorname{Re} \mu(\xi)<\operatorname{Re} \mu\left(\xi_{*}\right), \quad \xi \neq \pm \xi_{*}
$$

when $\varepsilon+\mathscr{O}\left(\varepsilon^{2}\right)>R\left(v^{2}-1\right)$.

Suppose $\varepsilon \geq 0$. Note that even though the spectrum is not completely complex, we can still apply Theorem 1. Indeed, the frequency $\xi_{*}$ equals

$$
\xi_{*}^{2}=v b^{2}\left(1-\frac{1+v}{2(1-v)} \frac{\varepsilon}{R}\right)+\mathscr{O}\left(\varepsilon^{2}\right)
$$

and clearly $\xi_{*}^{2}>\xi_{0}^{2}$. Then $\operatorname{Re} \mu(\xi)<\operatorname{Re} \mu\left(\xi_{*}\right)$ for all $\xi^{2}>\xi_{0}^{2}$, $\xi \neq \pm \xi_{*}$, provided that

$$
v^{2}<1+\frac{\varepsilon}{R}+\mathscr{O}\left(\varepsilon^{2}\right)<2-v^{2}<\frac{1}{v^{2}} .
$$

Under the same conditions, $\hat{Q}_{1,1}(\xi)-\hat{Q}_{2,2}(\xi)$ is growing on $\left(0, \xi_{0}\right)$ and

$$
\hat{Q}_{1,1}(\xi)-\hat{Q}_{2,2}(\xi) \leq 4 \psi^{\prime}(0) \varepsilon+\mathscr{O}\left(\varepsilon^{2}\right), \quad 0 \leq \xi<\xi_{0} .
$$

Using monotonicity properties we can also derive that

$$
\begin{aligned}
& \left(\hat{Q}_{1,1}(\xi)+\hat{Q}_{2,2}(\xi)\right)^{2}-4 \hat{Q}_{1,2}(\xi) \hat{Q}_{2,1}(\xi)< \\
& <\left(\hat{Q}_{1,1}(0)+\hat{Q}_{2,2}(0)\right)^{2}-4 \hat{Q}_{1,2}\left(\xi_{0}\right) \hat{Q}_{2,1}\left(\xi_{0}\right)= \\
& =\left(4 \psi^{\prime}(0) R\right)^{2} \frac{1+v^{2}}{1-v^{2}} \frac{\varepsilon}{R}+\mathscr{O}\left(\varepsilon^{2}\right)
\end{aligned}
$$

on $\left(0, \xi_{0}\right)$. We thus get an upper bound

$$
\mu(\xi)<2 \psi^{\prime}(0) R \sqrt{\frac{1+v^{2}}{1-v^{2}} \frac{\varepsilon}{R}}+\mathscr{O}(\varepsilon), \quad 0 \leq \xi<\xi_{0},
$$

which shows that $\mu(\xi)=\operatorname{Re} \mu(\xi)<\operatorname{Re} \mu\left(\xi_{*}\right)$ for $\xi^{2}<\xi_{0}^{2}$. As a result,

$$
\operatorname{Re} \mu(\xi)<\operatorname{Re} \mu\left(\xi_{*}\right), \quad \xi \neq \pm \xi_{*} .
$$

\section{B. Hopf bifurcation}

For the Hopf bifurcation to occur, we must have

$$
\mu\left(\xi_{*}\right)=\sigma_{*}+\mathrm{i} \omega_{*}, \quad \omega_{*} \neq 0 .
$$

Straightforward calculations show that

$$
\begin{aligned}
& \operatorname{Re} \mu\left(\xi_{*}\right)=\frac{\psi^{\prime}(0) R}{1+v}\left(1-v+\frac{\varepsilon}{R}\right)+\mathscr{O}\left(\varepsilon^{2}\right), \\
& \operatorname{Im} \mu\left(\xi_{*}\right)=\frac{\psi^{\prime}(0) R}{1+v}\left(\sqrt{(1-v)(3+v)}-\frac{\varepsilon}{R}\right)+\mathscr{O}\left(\varepsilon^{2}\right),
\end{aligned}
$$


whence we get the bifurcation condition

$$
\sigma_{*}=\frac{\psi^{\prime}(0) R}{1+v}\left(1-v+\frac{\varepsilon}{R}\right)+\mathscr{O}\left(\varepsilon^{2}\right) .
$$

In Figure 1, we show Hopf bifurcation curves in the $(R, v)$ parameter space for different values of $\sigma_{*} / \psi^{\prime}(0)$.

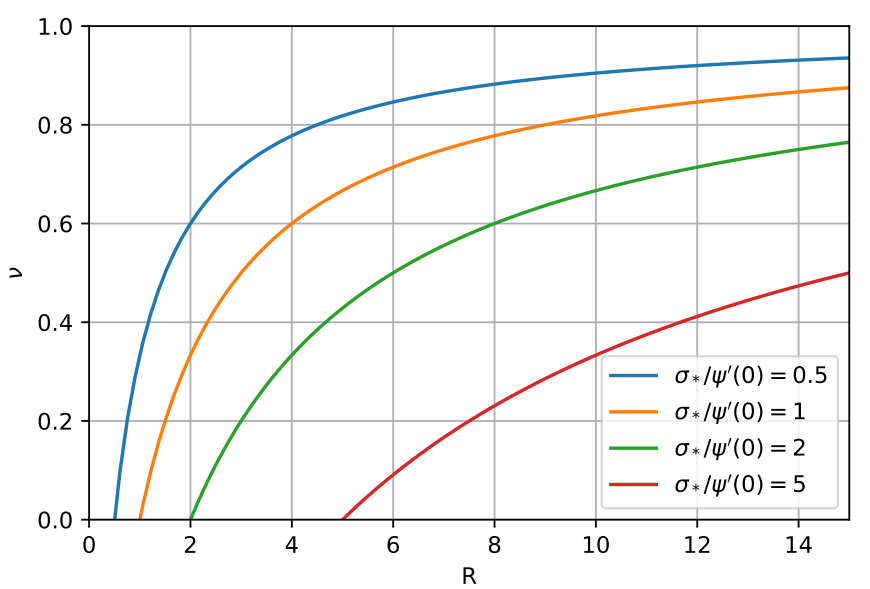

FIG. 1: Curves in the $(R, v)$ parameter space where Hopf bifurcation occurs for different values of $\sigma_{*} / \psi^{\prime}(0)$ and $\varepsilon=0$.

\section{Normal form}

Let $\sigma$ be a small perturbation of the critical parameter value $\sigma_{*}$. Then the real part of the velocity of the critical characteristic value is negative

$$
\operatorname{Re} \gamma=\left.\left(\frac{d}{d \sigma} \operatorname{Re} \lambda\left(\xi_{*}(0) ; \sigma\right)\right)\right|_{\sigma=0}=-1+\mathscr{O}\left(\varepsilon^{2}\right)<0
$$

and the condition $\sigma \operatorname{Re} \gamma>0$ transforms into $\sigma<0$.

Consider matrices $M_{k}(0)$. It follows from our derivations that

$$
\begin{aligned}
& M_{1}(0)=\left[\begin{array}{cc}
\psi^{\prime}(0)+\mathscr{O}\left(\varepsilon^{2}\right) & 0 \\
0 & -\psi^{\prime}(0)+\mathscr{O}\left(\varepsilon^{2}\right)
\end{array}\right], \\
& M_{2}(0)=\frac{1}{2}\left[\begin{array}{cc}
\psi^{\prime \prime \prime}(0) u_{0}+\mathscr{O}\left(\varepsilon^{2}\right) & 0 \\
0 & -\psi^{\prime \prime \prime}(0) v_{0}+\mathscr{O}\left(\varepsilon^{2}\right)
\end{array}\right], \\
& M_{3}(0)=\frac{1}{6}\left[\begin{array}{cc}
\psi^{\prime \prime \prime}(0)+\mathscr{O}\left(\varepsilon^{2}\right) & 0 \\
0 & -\psi^{\prime \prime \prime}(0)+\mathscr{O}\left(\varepsilon^{2}\right)
\end{array}\right] .
\end{aligned}
$$

In particular, $\left\|M_{2}(0)\right\|=\mathscr{O}(\varepsilon)$. Note also that

$$
\mathscr{P}_{1}=\left[\begin{array}{cc}
\frac{2 R}{1+v}\left(1+\frac{2-v}{2-2 v} \frac{\varepsilon}{R}\right) & \frac{2 R}{1+v}\left(1+\frac{v}{2-2 v} \frac{\varepsilon}{R}\right) \\
\frac{2 R}{1+v}\left(1+\frac{v}{2-2 v} \frac{\varepsilon}{R}\right) & \frac{2 R v}{1+v}\left(1+\frac{1}{2-2 v} \frac{\varepsilon}{R}\right)
\end{array}\right]+\mathscr{O}\left(\varepsilon^{2}\right) .
$$

Then the eigenvector $\zeta_{1}$ equals

$\zeta_{1}=\frac{\psi^{\prime}(0) R}{1+v}\left[\begin{array}{c}2+\frac{v}{1-v} \frac{\varepsilon}{R} \\ 1+v-\mathrm{i} \sqrt{(1-v)(3+v)}+\left(\frac{1}{1-v}-\mathrm{i}\right) \frac{\varepsilon}{R}\end{array}\right]+\mathscr{O}\left(\varepsilon^{2}\right)$
For simplicity, we will only write the $\mathscr{O}(1)$ terms in what follows. In that case, we have

$$
\zeta_{1}^{*}=\frac{1+v}{\psi^{\prime}(0) R}\left[\begin{array}{c}
\frac{1}{4}+\frac{i}{4} \frac{1+v}{\sqrt{(1-v)(3+v)}} \\
-\frac{i}{2} \frac{1}{\sqrt{(1-v)(3+v)}}
\end{array}\right]+\mathscr{O}(\varepsilon) .
$$

As $\left\|\zeta_{1}\right\|=\mathscr{O}(1)$ and $\left\|M_{2}(0)\right\|=\mathscr{O}(\varepsilon)$, the vectors $h_{j}, j=$ $1, \ldots, 5$ from Appendix A are all small, $\left\|h_{j}\right\|=\mathscr{O}(\varepsilon)$. Based on the property of the Hadamard product,

$$
\|x \odot y\| \leq\|x\|\|y\|,
$$

we can conclude that

$$
\begin{array}{rlrl}
\left\|M_{2}(0)\left(\overline{\zeta_{1}} \odot h_{1}\right)\right\| & =\mathscr{O}\left(\varepsilon^{2}\right), & & \left\|M_{2}(0)\left(\zeta_{1} \odot h_{2}\right)\right\|=\mathscr{O}\left(\varepsilon^{2}\right), \\
\left\|M_{2}(0)\left(\zeta_{1} \odot h_{3}\right)\right\| & =\mathscr{O}\left(\varepsilon^{2}\right), & \left\|M_{2}(0)\left(\overline{\zeta_{1}} \odot h_{4}\right)\right\|=\mathscr{O}\left(\varepsilon^{2}\right), \\
\left\|M_{2}(0)\left(\zeta_{1} \odot h_{5}\right)\right\| & =\mathscr{O}\left(\varepsilon^{2}\right) . &
\end{array}
$$

As a consequence, the normal form coefficients take a much simpler form:

$$
\begin{aligned}
& c_{1}=\frac{1}{2 l_{*}}\left\langle M_{3}(0)\left(\zeta_{1} \odot \zeta_{1} \odot \overline{\zeta_{1}}\right), \mathscr{P}_{1}^{T} \zeta_{1}^{*}\right\rangle+\mathscr{O}\left(\varepsilon^{2}\right), \\
& c_{2}=\frac{1}{l_{*}}\left\langle M_{3}(0)\left(\zeta_{1} \odot \overline{\zeta_{1}} \odot \zeta_{1}\right), \mathscr{P}_{1}^{T} \zeta_{1}^{*}\right\rangle+\mathscr{O}\left(\varepsilon^{2}\right) .
\end{aligned}
$$

Recall that for periodic traveling waves to be asymptotically stable, three conditions need to be met:

$$
\operatorname{Re} c_{1}<0, \quad \operatorname{Re}\left(c_{1}+c_{2}\right)<0, \quad \operatorname{Re}\left(c_{1}-c_{2}\right)>0 .
$$

In our case we have

$$
\begin{aligned}
& \operatorname{Re}\left(c_{1}+c_{2}\right)=3 \operatorname{Re} c_{1}+\mathscr{O}\left(\varepsilon^{2}\right), \\
& \operatorname{Re}\left(c_{1}-c_{2}\right)=-\operatorname{Re} c_{1}+\mathscr{O}\left(\varepsilon^{2}\right) .
\end{aligned}
$$

This means that the stability of periodic traveling waves depends on a single condition

$$
\operatorname{Re} c_{1}<0
$$

Direct calculations give

$c_{1}=\frac{1}{3 l_{*}}\left(\frac{\psi^{\prime}(0) R}{1+v}\right)^{3} \frac{\psi^{\prime \prime \prime}(0)}{\psi^{\prime}(0)}(1-v+\mathrm{i} \sqrt{(1-v)(3+v)})+\mathscr{O}(\varepsilon)$.

Therefore, since $v<1, \psi^{\prime}(0)>0$, and $\psi^{\prime \prime \prime}(0)<0$, the real part is negative,

$$
\operatorname{Re} c_{1}=\frac{1}{3 l_{*}}\left(\frac{\psi^{\prime}(0) R}{1+v}\right)^{3} \frac{\psi^{\prime \prime \prime}(0)}{\psi^{\prime}(0)}(1-v)+\mathscr{O}(\varepsilon)<0,
$$

. and periodic traveling waves are asymptotically stable for all parameters of this class as long as $|\mathcal{E}|$ is not too large. 
TABLE II: Example of parameters.

\begin{tabular}{ccccccccc}
\hline \hline$a_{11}$ & $a_{12}$ & $a_{21}$ & $a_{22}$ & $b_{11}$ & $b_{12}$ & $b_{21}$ & $b_{22}$ & $\sigma_{*}$ \\
\hline 3.05 & 3.00 & 3.00 & 0.30 & 1.00 & 1.00 & 1.00 & 0.10 & 1.00 \\
\hline \hline
\end{tabular}

\section{NUMERICAL EXAMPLES}

For the numerical examples, we choose $\psi_{1}=\psi_{2}=\psi$ with

$$
\psi(u)=\frac{2}{\pi} \arctan (0.6782 u)+1 .
$$

Consider the parameters from Table II. The constant solution is $u_{0}=0.404, v_{0}=0.287$. Upon linearization about $\left(u_{0}, v_{0}\right)$ we can find that the critical spatial frequency is $\xi_{*}=0.318$ and that $\lambda\left(\xi_{*}\right)= \pm 1.86 \mathrm{i}$ (see Figure 2). The computation of

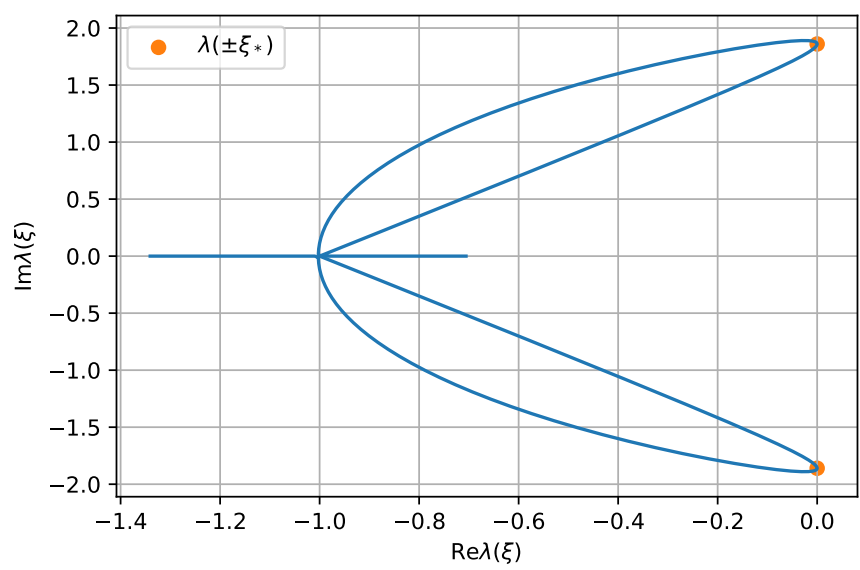

FIG. 2: Distribution of characteristic values $\lambda(\xi)$ on the complex plain for the parameters from Table II.

the normal form gives

$$
c_{1} \approx-0.0182-0.0386 \mathrm{i}, \quad c_{2} \approx-0.0365-0.0773 \mathrm{i},
$$

which means that the periodic traveling waves are asymptotically stable.

For numerical verification, we ran numerical simulations on the interval $\left(0,2 \pi / \xi_{*}\right)$ with periodic boundary conditions. We computed the convolutions using the fast Fourier transform and the convolution theorem.

In Figure 3, we present numerical simulations for box-like initial states:

$$
u_{0}(x)=2 \chi\left\{x<\pi / \xi_{*}\right\}-1, \quad v_{0}(x)=2 \chi\left\{x<\pi / \xi_{*}\right\}-1 .
$$

Here, $\chi\{\Omega\}$ is the indicator function of the set $\Omega$ such that $\chi\{\Omega\}(x)=1$ if $x \in \Omega$ and $\chi\{\Omega\}(x)=0$ if $x \notin \Omega$. In the formulas above, both $u_{0}$ and $v_{0}$ are equal to 1 on $\left(0, \pi / \xi_{*}\right)$ and to -1 on $\left(\pi / \xi_{*}, 2 \pi / \xi_{*}\right)$. As the simulations show, the solution retains the symmetry of the initial state and becomes a standing wave. However, if we shrink or enlarge the positive half to $\left(0, \pi / \xi_{*} \pm \varepsilon\right)$ for one of the components (let it be $\left.v_{0}\right)$, the solution turns into a periodic traveling wave since the initial state breaks the reflection symmetry of the system.

It is also important to understand how the system evolves from zero initial state in the presence of a forcing term. Namely, we are interested in time-oscillating point sources like

$$
f(x, t)=\delta\left(x-x_{0}\right) \sin (t),
$$

where $\delta(x)$ is the $\delta$-function. So we want to solve the following initial value problem:

$$
\begin{aligned}
& \frac{\partial u}{\partial t}=P_{1,1} * \psi_{1}(u)-P_{1,2} * \psi_{2}(v)-\sigma u+f_{1}(x, t), \\
& \frac{\partial v}{\partial t}=P_{2,1} * \psi_{1}(u)-P_{2,2} * \psi_{2}(v)-\sigma v+f_{2}(x, t), \\
& u(x, 0)=0, \quad v(x, 0)=0 .
\end{aligned}
$$

In Figure 4, we use

$$
f_{j}(x, t)=0.1 \delta\left(x-x_{j}\right) \sin (\omega t), \quad j=1,2,
$$

as the forcing terms. If $x_{1}=x_{2}$, that is the point sources are placed at the exactly same positions, the zero initial state evolves into a standing wave. Once again, this is because the reflection symmetry is not broken. Meanwhile, should the point sources be placed at different (possibly very close) positions, $x_{1} \neq x_{2}$, the solution is a periodic traveling wave, which propagates in the direction determined by the relative position of the two sources.

In Figure 5, we explore the case where the forcing terms have different oscillation frequencies:

$$
\begin{aligned}
& f_{1}(x, t)=0.1 \delta\left(x-\pi / 2 \xi_{*}\right) \sin (A t), \\
& f_{2}(x, t)=0.1 \delta(x) \sin (t) .
\end{aligned}
$$

We observed that for a fixed distance between the forcing terms, the propagation direction of the traveling waves depends on the ratio of the forcing term frequencies $A>0$. Namely, there is a threshold value $A_{*}$ such that waves propagate in different directions for $A<A_{*}$ and $A>A_{*}$. Moreover, as $A \rightarrow A_{*}$, the solution spends more time in the form of a standing wave, which suggests that at $A=A_{*}$ the solution is a standing wave.

\section{DISCUSSION}

With the development of modern technologies it has been possible to demonstrate the existence of periodic traveling waves in cortex during many human activities ${ }^{7}$. These propagating waves help explain brain dynamics observed at the population level ${ }^{30}$. For example, Huang et al. ${ }^{31,32}$ studied brain wave dynamics for years using voltage-sensitive dye imaging and demonstrated the presence of spiral dynamics in the intact mammalian brain during pharmacologically induced oscillations as well as during sleep-like states. These authors showed that traveling waves are emergent properties which can influence not only brain oscillation frequencies and spatial coherence but they can also modulate their amplitude. As a result 


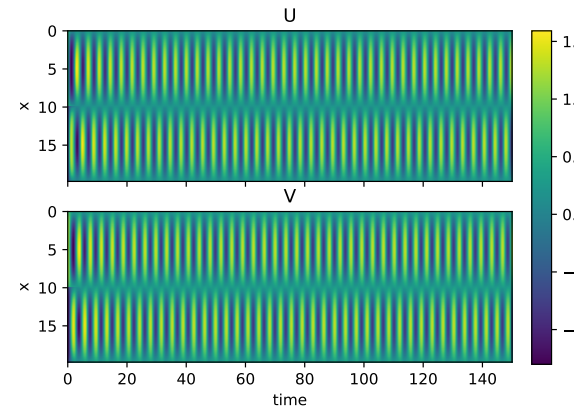

(a) $v_{0}(x)=2 \chi\left\{x<\pi / \xi_{*}\right\}-1$
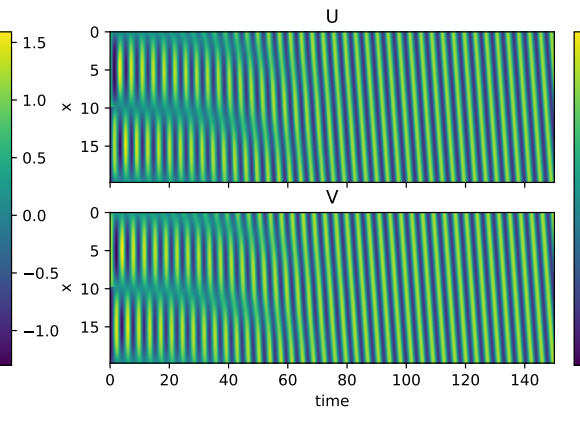

(b) $v_{0}(x)=2 \chi\left\{x<\pi / \xi_{*}-\varepsilon\right\}-1$

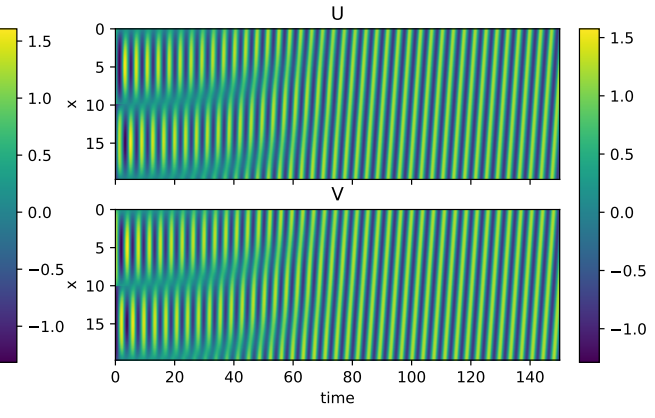

(c) $v_{0}(x)=2 \chi\left\{x<\pi / \xi_{*}+\varepsilon\right\}-1$

FIG. 3: Numerical simulation with the initial state $u_{0}(x)=2 \chi\left\{x<\pi / \xi_{*}\right\}-1$ and $\sigma=0.95$. Level lines of the solution $u(x, t)$ (upper row) and $v(x, t)$ (lower row) are shown for three different initial states $v_{0}(x)$. Standing waves are observed for the symmetric initial state (a), periodic traveling waves for the asymmetric initial states $(b, c)$.

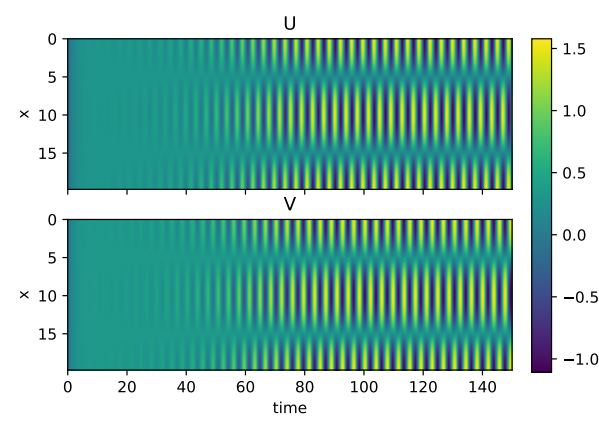

(a) $x_{1}=0, x_{2}=0$

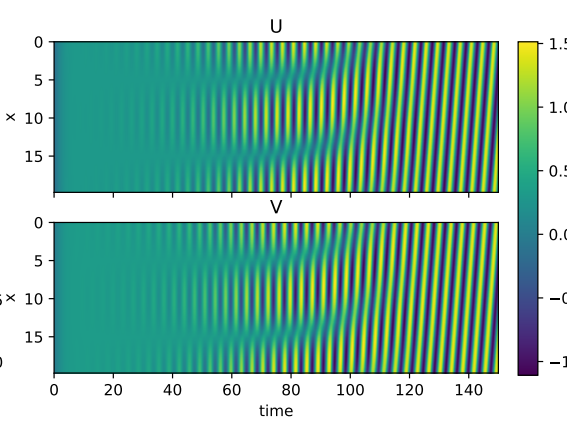

(b) $x_{1}=\varepsilon, x_{2}=0$

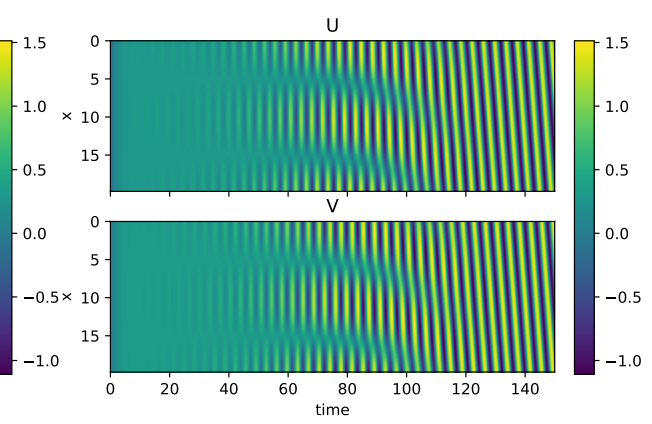

(c) $x_{1}=0, x_{2}=\varepsilon$

FIG. 4: Numerical simulation with the forcing terms $f_{j}(x, t)=0.1 \delta\left(x-x_{j}\right) \sin (\omega t), j=1,2$, and $\sigma=0.92$. Level lines of the solution $u(x, t)$ (upper row) and $v(x, t)$ (lower row) are shown for three different forcing terms. Standing waves are observed for the symmetric forcing term (a), periodic traveling waves for the asymmetric forcing terms $(b, c)$.

these waves «may contribute to both normal cortical processing and to pathological patterns of activity such as those found in epilepsy» (Ref. 30, P. 1591). Interestingly Stead et al. ${ }^{33}$ observed «microseizures» (spiral waves) on isolated research microelectrodes in both epileptic patients (in regions prone to generate epileptic seizures) but also in control subjects. They conclude that only «the density of these phenomena distinguishes normal from epileptic brain» (Ref. 33, P. 2789).

Although the functional role of traveling waves is not completely elucidated yet, new techniques based on targeted neuromodulation of traveling waves using electric fields are already being explored as potential therapeutic strategies. For example, Whalen et al. ${ }^{2}$ were able to control the modulation, suppression and prevention or to increase propagation velocity of spreading depression with DC electrical fields in brain slices thus demonstrating «the potential feasibility of electrical control and prevention of spreading depression» (Ref. 2, P. 8769). Using coupled oscillators Ermentrout and Kleinfeld ${ }^{34}$ also studied electrical waves in the cortex and speculated as to their computational role.

Neural field models provide an appropriate tool to study these waves but the results differ for different models. A single equation with symmetric connectivity functions for activating and inhibitory signals does not have solutions in the form of periodic traveling waves. In the case of asymmetric functions, such solutions exist, they are stable, and solutions with different frequencies (speeds) are observed for the same values of parameters ${ }^{35}$. The last property is important for the understanding of the existence of the waves with different frequencies during brain activity.

A single equation with symmetric connectivity functions can also manifest periodic waves in the presence of time delay $^{36}$. The periodic waves bifurcate from the stationary, spatially homogeneous solution being unstable, and they become stable if time delay exceeds some critical value. As before, the coexistence of waves with different frequencies is observed. Another important characterization of periodic waves related to the choice of initial conditions should also be taken into account. We will return to this question below.

The models with two different neuron populations, excitatory and inhibitory, can also describe the propagation of periodic waves ${ }^{17,18,21,22,28}$. In this work we considered a particular case of the model Ref. 28 without time delay and with symmetric connectivity functions. Nonlinear bifurcation analysis shows two types of solutions: standing oscillating solutions with the behavior qualitatively similar to the product of two periodic functions, $\cos (\omega t) \cos (k x)$, and periodic traveling waves similar to $\cos (\omega t+k x)$. Their stability depends on 


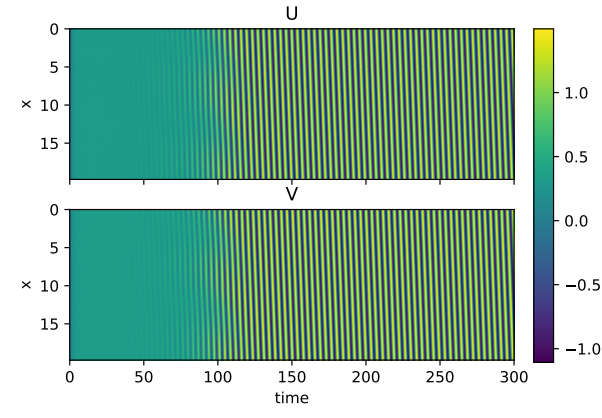

(a) $A=1$

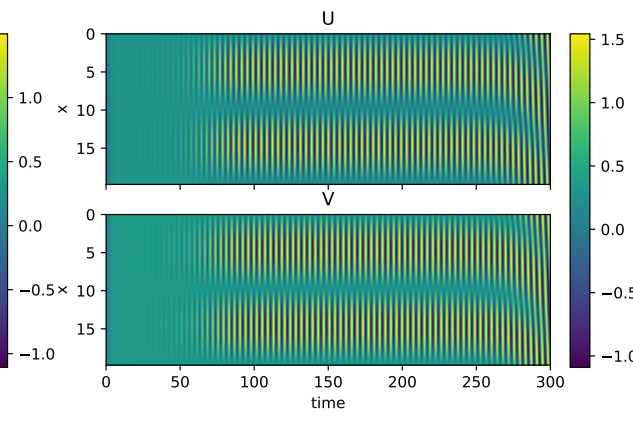

(b) $A=1.973469$

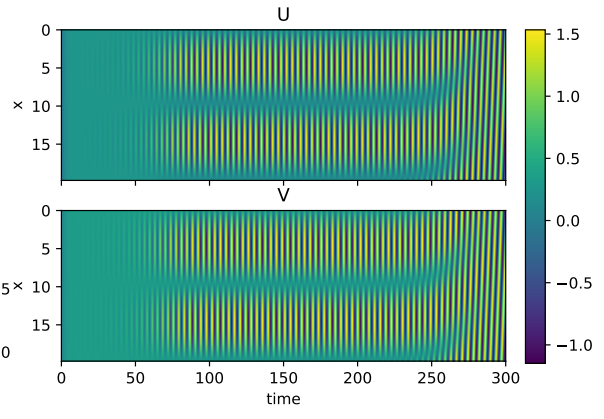

(c) $A=1.973470$

FIG. 5: Numerical simulation with the forcing terms $f_{1}(x, t)=0.1 \delta\left(x-\pi / 2 \xi_{*}\right) \sin (A t), f_{2}(x, t)=0.1 \delta(x) \sin (t)$, and $\sigma=0.92$. Level lines of the solution $u(x, t)$ (upper row) and $v(x, t)$ (lower row) are shown for three different forcing terms. The traveling waves move to the right for $A$ below the threshold value $(a, b)$ and to the left for $A$ above the threshold value (c).

the values of parameters, and they are mutually exclusive.

From the biological point of view we are interested not only in the existence and stability of periodic traveling waves but also in their initiation, that is, under what conditions (initial, boundary, etc), the solution will approach the wave. This question can be potentially important to determine the role of epicenters (hubs) in the functioning of brain connectome. The epicenters receive the information from other epicenters or from the external body organs, they process this information and send it further downstream. Assuming that the synchronization of epicenters is effectuated by cortical waves, we can suppose that the waves are initiated locally by the activated epicenter. In the case of a single equation, such initiation appears to be possible for asymmetric connectivity function but not for symmetric connectivity functions and time delay ${ }^{36}$. The latter requires the initial conditions close to the wave on the whole space interval. The model considered in this work admits wave initiation by local forcing terms (additional terms in the equations). If the forcing term is symmetric, then standing oscillating solutions are observed. In the case of asymmetric forcing term, under the parameters for which the periodic wave is stable, solution converges to the wave.

Let us note that the direction of propagation is one of the important parameters characterizing cortical waves. We show that this direction is determined by the forcing term. In the 2D setting we expect that we can obtain any direction between 0 and $2 \pi$. Hence, the epicenters can exchange directed signals ${ }^{12}$. Wave initiation by local forcing terms is observed in the case of brain stimulation with electrodes ${ }^{37}$. External brain stimulation can be used in order to restore wave propagation in a partially damaged tissue ${ }^{35,36}$. Recent perspectives propose to control brain traveling waves in human subjects by stimulating preidentified nodes using electrical stimulation. One of these initiatives propose to modulate the activity of cortical epicenters of the language network to treat chronic conditions such as post stroke aphasia ${ }^{38,39}$.

\section{ACKNOWLEDGMENTS}

Funding was provided by "RUDN University Program 5-100" (SB, implementation of the model; VV, designed the model and supervised analyses and interpretations), by CorStim (AB, designed and conceptualized the research), by the Moscow Center for Fundamental and Applied Mathematics (SB, calculations; agreement with the Ministry of Education and Science of the Russian Federation No. 075-15-20191624). The manuscript was written and revised with input from all three authors.

\section{DATA AVAILABILITY}

Data sharing is not applicable to this article as no new data were created or analyzed in this study.

\section{Appendix A: Computation of the normal form coefficients}

To compute the cubic coefficients $c_{1} \in \mathbb{C}$ and $c_{2} \in \mathbb{C}$, we introduce a function

$$
\tilde{F}(w, \sigma)=F(w, \sigma)+L(\sigma) w-L(0) w-\sigma L^{\prime}(0) w
$$

and use the formulas given in Ref. 29. The computation involves second and third-order Fréchet derivatives of $\tilde{F}(w, \sigma)$ $\left(\tilde{F}_{w w}(0,0)\right.$ and $\left.\tilde{F}_{w w w}(0,0)\right)$ at $w=0$ and $\sigma=0$ :

$$
\begin{aligned}
& \tilde{F}_{2}\left(w_{1}, w_{2}\right)=\sum_{n \in \mathbb{Z}} \mathscr{P}_{n} M_{2}(0)\left(w_{1} \odot w_{2}\right)_{n} e_{n}, \\
& \tilde{F}_{3}\left(w_{1}, w_{2}, w_{3}\right)=\sum_{n \in \mathbb{Z}} \mathscr{P}_{n} M_{3}(0)\left(w_{1} \odot w_{2} \odot w_{3}\right)_{n} e_{n} .
\end{aligned}
$$


It remains to evaluate the following expressions:

$$
\begin{aligned}
& g_{1}=\frac{1}{2}\left(2 \mathrm{i} \omega_{*}-L(0)\right)^{-1} \tilde{F}_{2}\left(q_{1}, q_{1}\right), \\
& g_{2}=-L(0)^{-1} \tilde{F}_{2}\left(\overline{q_{1}}, q_{1}\right), \\
& g_{3}=\left(2 \mathrm{i} \omega_{*}-L(0)\right)^{-1} \tilde{F}_{2}\left(q_{1}, q_{3}\right), \\
& g_{4}=-L(0)^{-1} \tilde{F}_{2}\left(q_{1}, \overline{q_{3}}\right), \\
& g_{5}=-L(0)^{-1} \tilde{F}_{2}\left(q_{3}, \overline{q_{3}}\right) .
\end{aligned}
$$

We treat them one by one:

1.

$$
\begin{aligned}
& \tilde{F}_{2}\left(q_{1}, q_{1}\right)=\frac{1}{\sqrt{l_{*}}} \mathscr{P}_{2} M_{2}(0)\left(\zeta_{1} \odot \zeta_{1}\right) e_{2}, \\
& g_{1}=h_{1} e_{2}, \quad h_{1} \in \mathbb{C}^{2}, \\
& \left(2 \mathrm{i} \omega_{*}-\mathscr{P}_{2} M_{1}(0)\right) h_{1}=\frac{1}{2 \sqrt{l_{*}}} \mathscr{P}_{2} M_{2}(0)\left(\zeta_{1} \odot \zeta_{1}\right) ;
\end{aligned}
$$

2.

$$
\begin{aligned}
& \tilde{F}_{2}\left(\overline{q_{1}}, q_{1}\right)=\frac{1}{\sqrt{l_{*}}} \mathscr{P}_{0} M_{2}(0)\left(\overline{\zeta_{1}} \odot \zeta_{1}\right) e_{0}, \\
& g_{2}=h_{2} e_{0}, \quad h_{2} \in \mathbb{C}^{2}, \\
& \left(\mathscr{P}_{0} M_{1}(0)\right) h_{2}=-\frac{1}{\sqrt{l_{*}}} \mathscr{P}_{0} M_{2}(0)\left(\overline{\zeta_{1}} \odot \zeta_{1}\right) ;
\end{aligned}
$$

3.

$$
\begin{aligned}
& \tilde{F}_{2}\left(q_{1}, q_{3}\right)=\frac{1}{\sqrt{l_{*}}} \mathscr{P}_{2} M_{2}(0)\left(\zeta_{1} \odot \overline{\zeta_{1}}\right) e_{2}, \\
& g_{3}=h_{3} e_{2}, \quad h_{3} \in \mathbb{C}^{2}, \\
& \left(2 \mathrm{i} \omega_{*}-\mathscr{P}_{2} M_{1}(0)\right) h_{3}=\frac{1}{\sqrt{l_{*}}} \mathscr{P}_{2} M_{2}(0)\left(\zeta_{1} \odot \overline{\zeta_{1}}\right) ;
\end{aligned}
$$

4.

$$
\begin{aligned}
& \tilde{F}_{2}\left(q_{1}, \overline{q_{3}}\right)=\frac{1}{\sqrt{l_{*}}} \mathscr{P}_{0} M_{2}(0)\left(\zeta_{1} \odot \zeta_{1}\right) e_{0}, \\
& g_{4}=h_{4} e_{0}, \quad h_{4} \in \mathbb{C}^{2}, \\
& \left(\mathscr{P}_{0} M_{1}(0)\right) h_{4}=-\frac{1}{\sqrt{l_{*}}} \mathscr{P}_{0} M_{2}(0)\left(\zeta_{1} \odot \zeta_{1}\right) ;
\end{aligned}
$$

5.

$$
\begin{aligned}
& \tilde{F}_{2}\left(q_{3}, \overline{q_{3}}\right)=\frac{1}{\sqrt{l_{*}}} \mathscr{P}_{0} M_{2}(0)\left(\overline{\zeta_{1}} \odot \zeta_{1}\right) e_{0}, \\
& g_{5}=h_{5} e_{0}, \quad h_{5} \in \mathbb{C}^{2}, \\
& \left(\mathscr{P}_{0} M_{1}(0)\right) h_{5}=-\frac{1}{\sqrt{l_{*}}} \mathscr{P}_{0} M_{2}(0)\left(\overline{\zeta_{1}} \odot \zeta_{1}\right) .
\end{aligned}
$$

We also need two terms with third-order derivatives:

$$
\begin{aligned}
& \tilde{F}_{3}\left(q_{1}, q_{1}, \overline{q_{1}}\right)=\frac{1}{l_{*}} \mathscr{P}_{1} M_{3}(0)\left(\zeta_{1} \odot \zeta_{1} \odot \overline{\zeta_{1}}\right) e_{1}, \\
& \tilde{F}_{3}\left(q_{1}, q_{3}, \overline{q_{3}}\right)=\frac{1}{l_{*}} \mathscr{P}_{1} M_{3}(0)\left(\zeta_{1} \odot \overline{\zeta_{1}} \odot \zeta_{1}\right) e_{1} .
\end{aligned}
$$

The normal form coefficients are then given by the following formulas ${ }^{29}$ :

$c_{1}=\frac{1}{2}\left\langle\tilde{F}_{3}\left(q_{1}, q_{1}, \overline{q_{1}}\right)+2 \tilde{F}_{2}\left(\overline{q_{1}}, g_{1}\right)+2 \tilde{F}_{2}\left(q_{1}, g_{2}\right), q_{1}^{*}\right\rangle_{X}$, $c_{2}=\left\langle\tilde{F}_{3}\left(q_{1}, q_{3}, \overline{q_{3}}\right)+\tilde{F}_{2}\left(\overline{q_{3}}, g_{3}\right)+\tilde{F}_{2}\left(q_{3}, g_{4}\right)+\tilde{F}_{2}\left(q_{1}, g_{5}\right), q_{1}^{*}\right\rangle_{X}$.

It follows that

$$
\begin{aligned}
c_{1} & =\left\langle\frac{1}{2 l_{*}} \mathscr{P}_{1} M_{3}(0)\left(\zeta_{1} \odot \zeta_{1} \odot \overline{\zeta_{1}}\right)+\frac{1}{\sqrt{l_{*}}} \mathscr{P}_{1} M_{2}(0)\left(\overline{\zeta_{1}} \odot h_{1}\right)+\frac{1}{\sqrt{l_{*}}} \mathscr{P}_{1} M_{2}(0)\left(\zeta_{1} \odot h_{2}\right), \zeta_{1}^{*}\right\rangle \\
= & \frac{1}{\sqrt{l_{*}}}\left\langle\frac{1}{2 \sqrt{l_{*}}} M_{3}(0)\left(\zeta_{1} \odot \zeta_{1} \odot \overline{\zeta_{1}}\right)+M_{2}(0)\left(\overline{\zeta_{1}} \odot h_{1}\right)+M_{2}(0)\left(\zeta_{1} \odot h_{2}\right), \mathscr{P}_{1}^{T} \zeta_{1}^{*}\right\rangle, \\
c_{2}= & \left\langle\frac{1}{l_{*}} \mathscr{P}_{1} M_{3}(0)\left(\zeta_{1} \odot \overline{\zeta_{1}} \odot \zeta_{1}\right)+\frac{1}{\sqrt{l_{*}}} \mathscr{P}_{1} M_{2}(0)\left(\zeta_{1} \odot h_{3}\right)+\frac{1}{\sqrt{l_{*}}} \mathscr{P}_{1} M_{2}(0)\left(\overline{\zeta_{1}} \odot h_{4}\right)+\frac{1}{\sqrt{l_{*}}} \mathscr{P}_{1} M_{2}(0)\left(\zeta_{1} \odot h_{5}\right), \zeta_{1}^{*}\right\rangle \\
= & \frac{1}{\sqrt{l_{*}}}\left\langle\frac{1}{\sqrt{l_{*}}} M_{3}(0)\left(\zeta_{1} \odot \overline{\zeta_{1}} \odot \zeta_{1}\right)+M_{2}(0)\left(\zeta_{1} \odot h_{3}\right)+M_{2}(0)\left(\overline{\zeta_{1}} \odot h_{4}\right)+M_{2}(0)\left(\zeta_{1} \odot h_{5}\right), \mathscr{P}_{1}^{T} \zeta_{1}^{*}\right\rangle .
\end{aligned}
$$

${ }^{1}$ M. Massimini, R. Huber, F. Ferrarelli, S. Hill, and G. Tononi, "The Sleep Slow Oscillation as a Traveling Wave," Journal of Neuroscience 24, 68626870 (2004).
${ }^{2}$ A. J. Whalen, Y. Xiao, H. Kadji, M. A. Dahlem, B. J. Gluckman, and S. J. Schiff, "Control of Spreading Depression with Electrical Fields," Scientific Reports 8, 1-9 (2018). 
${ }^{3}$ N. Kopell and L. N. Howard, "Plane Wave Solutions to Reaction-Diffusion Equations," Studies in Applied Mathematics 52, 291-328 (1973).

${ }^{4}$ N. Kopell and L. N. Howard, "Horizontal Bands in the Belousov Reaction," Science 180, 1171-1173 (1973).

${ }^{5}$ A. Bahramisharif, M. A. J. van Gerven, E. J. Aarnoutse, M. R. Mercier, T. H. Schwartz, J. J. Foxe, N. F. Ramsey, and O. Jensen, "Propagating Neocortical Gamma Bursts Are Coordinated by Traveling Alpha Waves," Journal of Neuroscience 33, 18849-18854 (2013).

${ }^{6}$ M. Halgren, I. Ulbert, H. Bastuji, D. Fabó, L. Erőss, M. Rey, O. Devinsky, W. K. Doyle, R. Mak-McCully, E. Halgren, L. Wittner, P. Chauvel, G. Heit, E. Eskandar, A. Mandell, and S. S. Cash, "The generation and propagation of the human alpha rhythm," Proceedings of the National Academy of Sciences 116, 23772-23782 (2019).

${ }^{7}$ D. M. Alexander, A. R. Nikolaev, P. Jurica, M. Zvyagintsev, K. Mathiak, and C. van Leeuwen, "Global Neuromagnetic Cortical Fields Have NonZero Velocity,” PLoS ONE 11 (2016), 10.1371/journal.pone.0148413.

${ }^{8}$ J.-Y. Wu, X. Huang, and C. Zhang, "Propagating Waves of Activity in the Neocortex: What They Are, What They Do," The Neuroscientist 14, 487502 (2008)

${ }^{9}$ L. Muller, F. Chavane, J. Reynolds, and T. J. Sejnowski, "Cortical travelling waves: Mechanisms and computational principles," Nature Reviews Neuroscience 19, 255-268 (2018).

${ }^{10}$ H. Zhang, A. J. Watrous, A. Patel, and J. Jacobs, "Theta and Alpha Oscillations Are Traveling Waves in the Human Neocortex," Neuron 98, 12691281.e4 (2018)

${ }^{11} \mathrm{~J}$. Rapela, "Traveling waves appear and disappear in unison with produced speech," arXiv:1806.09559 [q-bio] (2018), arXiv:1806.09559 [q-bio]

${ }^{12}$ K. Takahashi, M. Saleh, R. D. Penn, and N. Hatsopoulos, "Propagating Waves in Human Motor Cortex," Frontiers in Human Neuroscience 5 (2011), 10.3389/fnhum.2011.00040.

${ }^{13}$ D. Lozano-Soldevilla and R. VanRullen, "The Hidden Spatial Dimension of Alpha: 10-Hz Perceptual Echoes Propagate as Periodic Traveling Waves in the Human Brain," Cell Reports 26, 374-380.e4 (2019).

${ }^{14}$ V. Botella-Soler, M. Valderrama, B. Crépon, V. Navarro, and M. Le Van Quyen, "Large-Scale Cortical Dynamics of Sleep Slow Waves," PLoS ONE 7 (2012), 10.1371/journal.pone.0030757.

${ }^{15} \mathrm{~J}$. Weaver, "How brain waves help us make sense of speech," PLoS Biology 11 (2013).

${ }^{16}$ D. Pinotsis, P. Robinson, P. beim Graben, and K. Friston, "Neural masses and fields: Modeling the dynamics of brain activity," Frontiers in Computational Neuroscience 8 (2014), 10.3389/fncom.2014.00149.

${ }^{17} \mathrm{H}$. R. Wilson and J. D. Cowan, "A mathematical theory of the functional dynamics of cortical and thalamic nervous tissue," Kybernetik 13, 55-80 (1973).

${ }^{18}$ S. Atasoy, I. Donnelly, and J. Pearson, "Human brain networks function in connectome-specific harmonic waves," Nature Communications 7, 1-10 (2016).

${ }^{19}$ S. Heitmann, P. Gong, and M. Breakspear, "A computational role for bistability and traveling waves in motor cortex," Frontiers in Computational Neuroscience 6 (2012), 10.3389/fncom.2012.00067.

${ }^{20}$ N. A. Venkov, S. Coombes, and P. C. Matthews, "Dynamic instabilities in scalar neural field equations with space-dependent delays," Physica D: Nonlinear Phenomena 232, 1-15 (2007)

${ }^{21}$ F. M. Atay and A. Hutt, "Neural Fields with Distributed Transmission Speeds and Long-Range Feedback Delays," SIAM Journal on Applied Dynamical Systems 5, 670-698 (2006).
${ }^{22}$ G. B. Ermentrout, S. E. Folias, and Z. P. Kilpatrick, "Spatiotemporal Pattern Formation in Neural Fields with Linear Adaptation," in Neural Fields: Theory and Applications, edited by S. Coombes, P. beim Graben, R. Potthast, and J. Wright (Springer, Berlin, Heidelberg, 2014) pp. 119-151.

${ }^{23}$ H. G. E. Meijer and S. Coombes, "Travelling waves in a neural field model with refractoriness," Journal of Mathematical Biology 68, 12491268 (2014).

${ }^{24}$ G. B. Ermentrout and J. B. McLeod, "Existence and uniqueness of travelling waves for a neural network," Proceedings of the Royal Society of Edinburgh Section A: Mathematics 123, 461-478 (1993/ed).

${ }^{25}$ Z. Chen, B. Ermentrout, and X.-J. Wang, "Wave Propagation Mediated by GABAB Synapse and Rebound Excitation in an Inhibitory Network: A Reduced Model Approach," Journal of Computational Neuroscience 5, 5369 (1998).

${ }^{26}$ D. J. Pinto and G. B. Ermentrout, "Spatially Structured Activity in Synaptically Coupled Neuronal Networks: I. Traveling Fronts and Pulses," SIAM Journal on Applied Mathematics 62, 206-225 (2001).

${ }^{27}$ D. J. Pinto and G. B. Ermentrout, "Spatially Structured Activity in Synaptically Coupled Neuronal Networks: II. Lateral Inhibition and Standing Pulses," SIAM Journal on Applied Mathematics 62, 226-243 (2001).

${ }^{28}$ J. Senk, K. Korvasová, J. Schuecker, E. Hagen, T. Tetzlaff, M. Diesmann, and M. Helias, "Conditions for wave trains in spiking neural networks," Physical Review Research 2, 023174 (2020).

${ }^{29}$ S. A. van Gils and J. Mallet-Paret, "Hopf bifurcation and symmetry: Travelling and standing waves on the circle," Proceedings of the Royal Society of Edinburgh: Section A Mathematics 104, 279-307 (1986).

${ }^{30}$ A. Keane and P. Gong, "Propagating Waves Can Explain Irregular Neural Dynamics," Journal of Neuroscience 35, 1591-1605 (2015).

${ }^{31}$ X. Huang, W. C. Troy, Q. Yang, H. Ma, C. R. Laing, S. J. Schiff, and J.Y. Wu, "Spiral Waves in Disinhibited Mammalian Neocortex," Journal of Neuroscience 24, 9897-9902 (2004).

${ }^{32}$ X. Huang, W. Xu, J. Liang, K. Takagaki, X. Gao, and J.-y. Wu, "Spiral Wave Dynamics in Neocortex," Neuron 68, 978-990 (2010).

${ }^{33}$ M. Stead, M. Bower, B. H. Brinkmann, K. Lee, W. R. Marsh, F. B. Meyer, B. Litt, J. Van Gompel, and G. A. Worrell, "Microseizures and the spatiotemporal scales of human partial epilepsy," Brain 133, 2789-2797 (2010).

${ }^{34}$ G. B. Ermentrout and D. Kleinfeld, "Traveling Electrical Waves in Cortex: Insights from Phase Dynamics and Speculation on a Computational Role,' Neuron 29, 33-44 (2001)

${ }^{35}$ N. Bessonov, A. Beuter, S. Trofimchuk, and V. Volpert, "Estimate of the travelling wave speed for an integro-differential equation," Applied Mathematics Letters 88, 103-110 (2019).

${ }^{36}$ N. Bessonov, A. Beuter, S. Trofimchuk, and V. Volpert, "Cortical waves and post-stroke brain stimulation," Mathematical Methods in the Applied Sciences 42, 3912-3928 (2019).

${ }^{37}$ A. Opitz, A. Falchier, C.-G. Yan, E. M. Yeagle, G. S. Linn, P. Megevand, A. Thielscher, R. D. A, M. P. Milham, A. D. Mehta, and C. E. Schroeder, "Spatiotemporal structure of intracranial electric fields induced by transcranial electric stimulation in humans and nonhuman primates," Scientific Reports 6, 1-11 (2016)

${ }^{38}$ A. Beuter, A. Balossier, S. Trofimchuk, and V. Volpert, "Modeling of poststroke stimulation of cortical tissue," Mathematical Biosciences 305, 146159 (2018).

${ }^{39}$ A. Beuter, A. Balossier, F. Vassal, S. Hemm, and V. Volpert, "Cortical stimulation in aphasia following ischemic stroke: Toward model-guided electrical neuromodulation,” Biological Cybernetics 114, 5-21 (2020). 


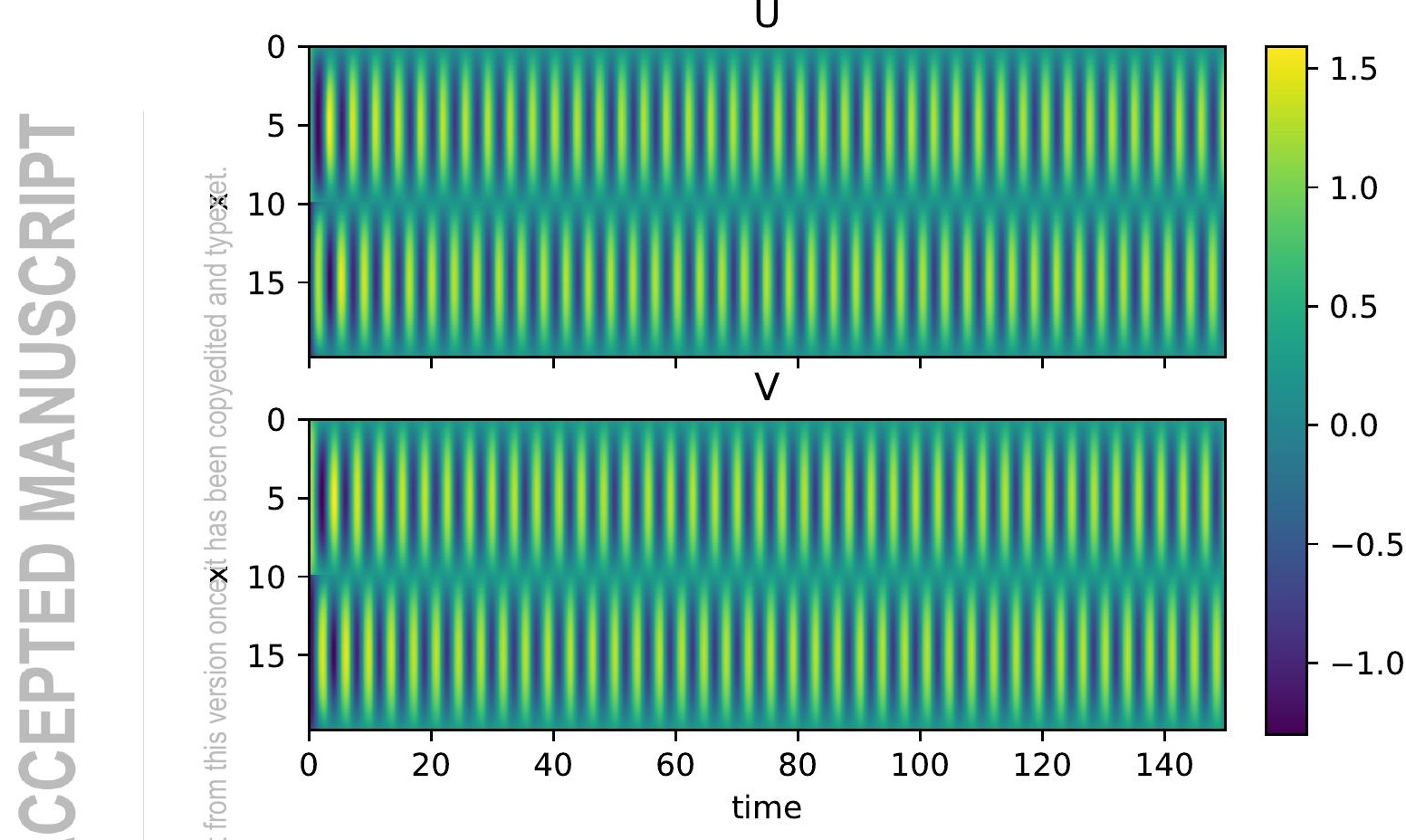

1
0
10

这

言

응

उ

잉

웅

등 흥

क

(1)

픈

뜰 믄

힐

오 빰

足皆

穵

랑

름

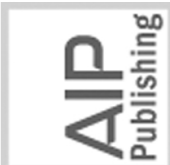




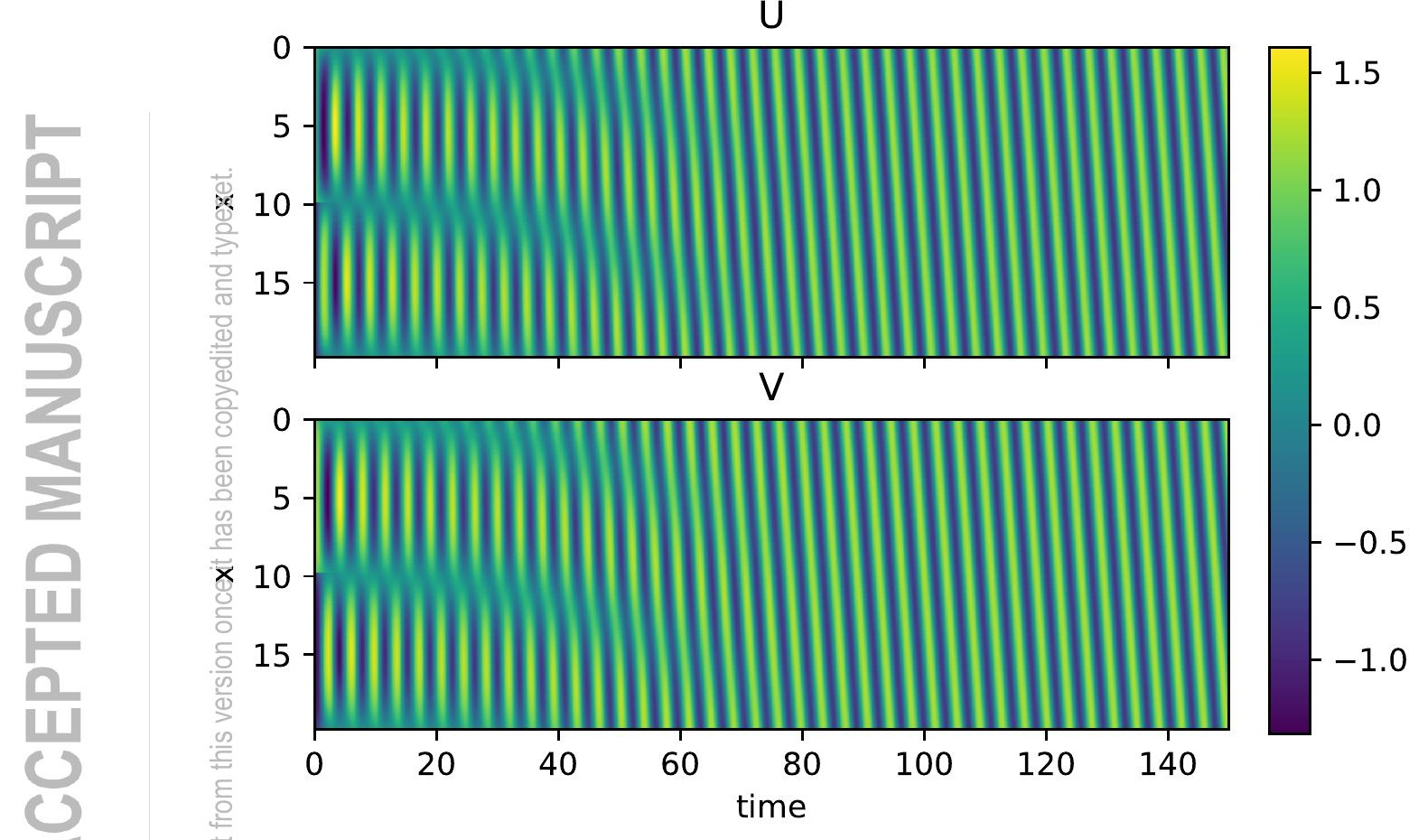

1
0
10

는 


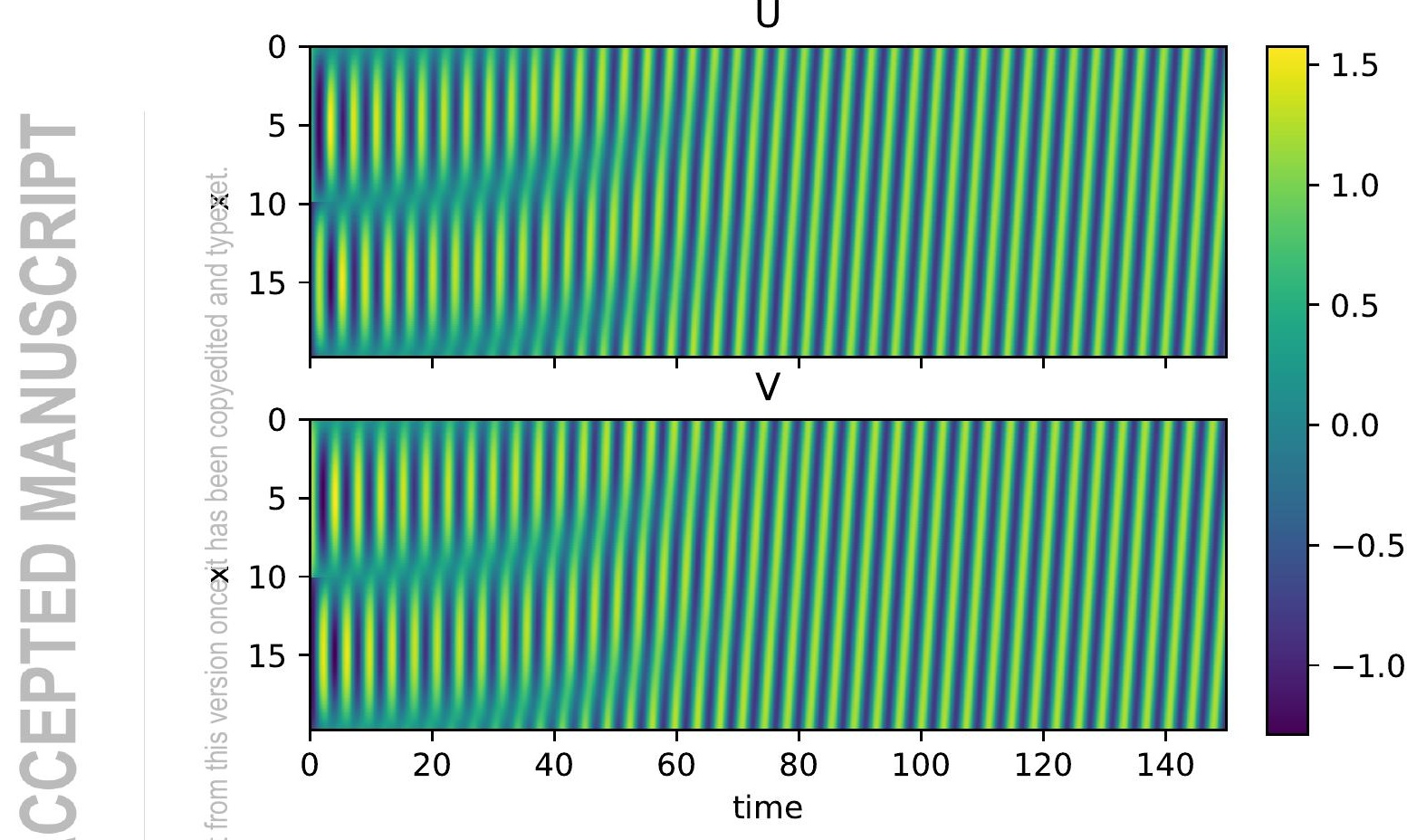

$\frac{1}{0}$

넌

事

응

낭

잉

웅

드음

क्ष

(1)

픈

땉 문

힘

호빰

는

穵

崖

름ㅁ

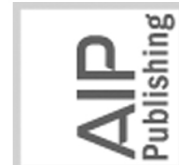




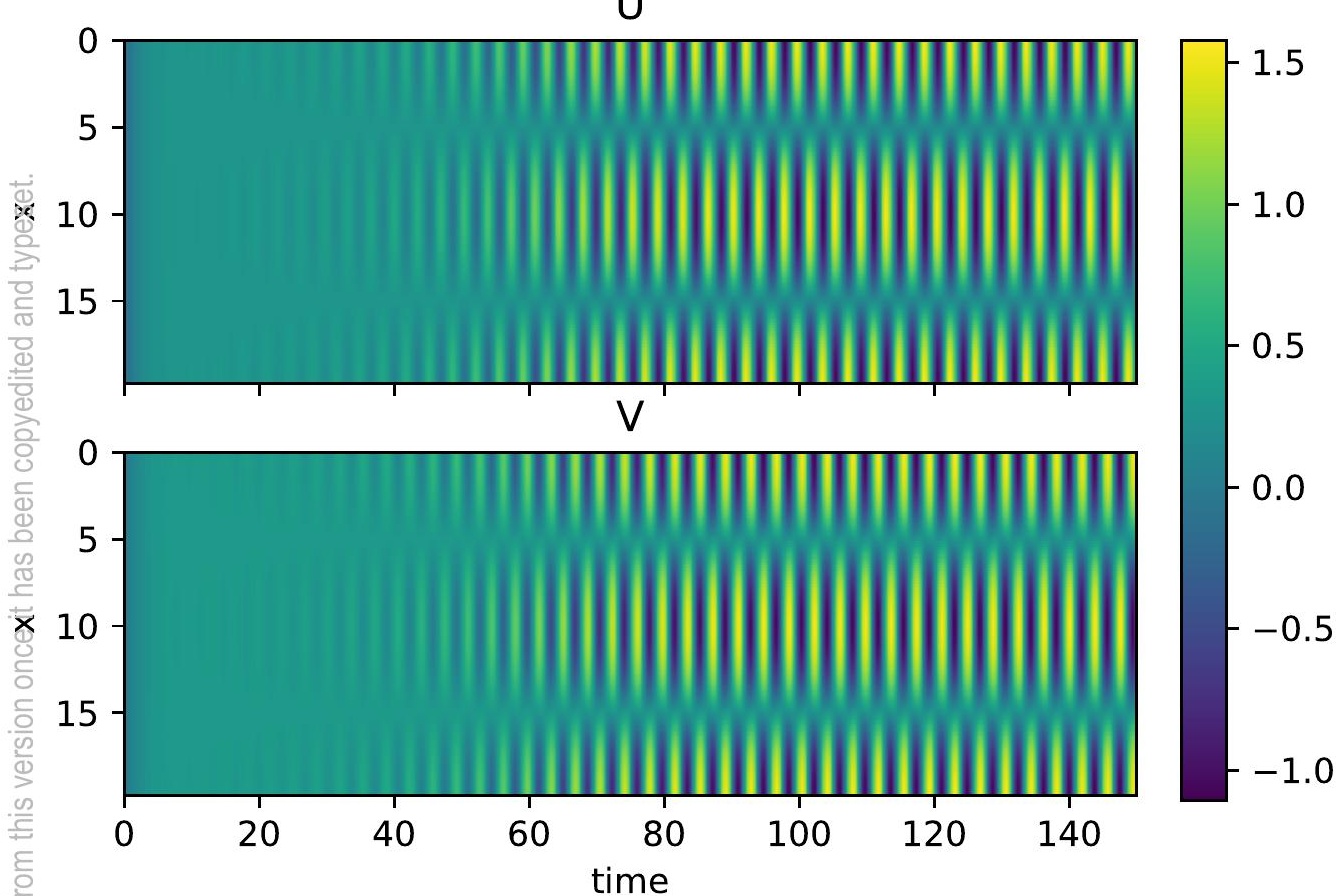




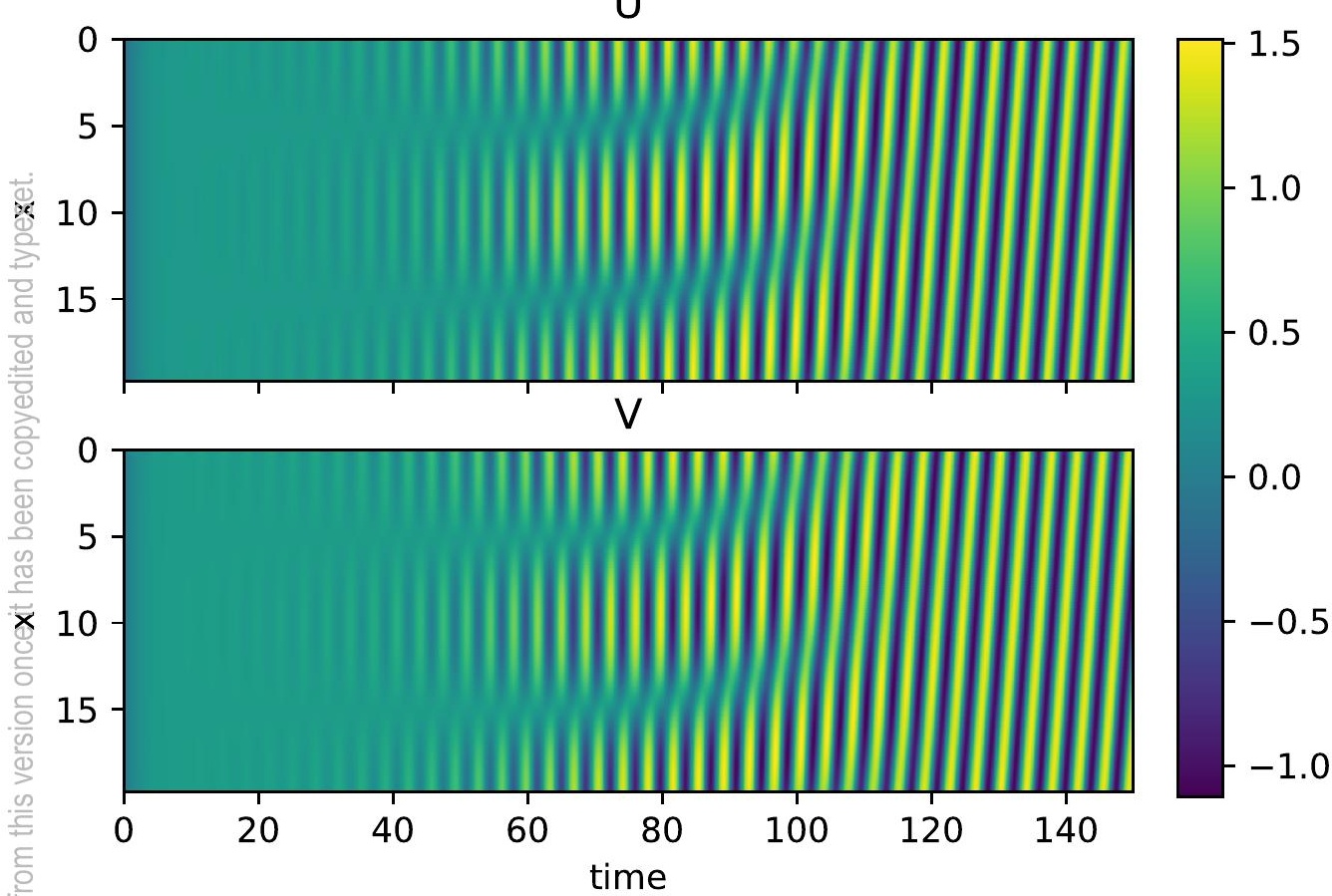

흔

क्ष

(1)

竞

을 만

힐

호빰

芒声

它

를 


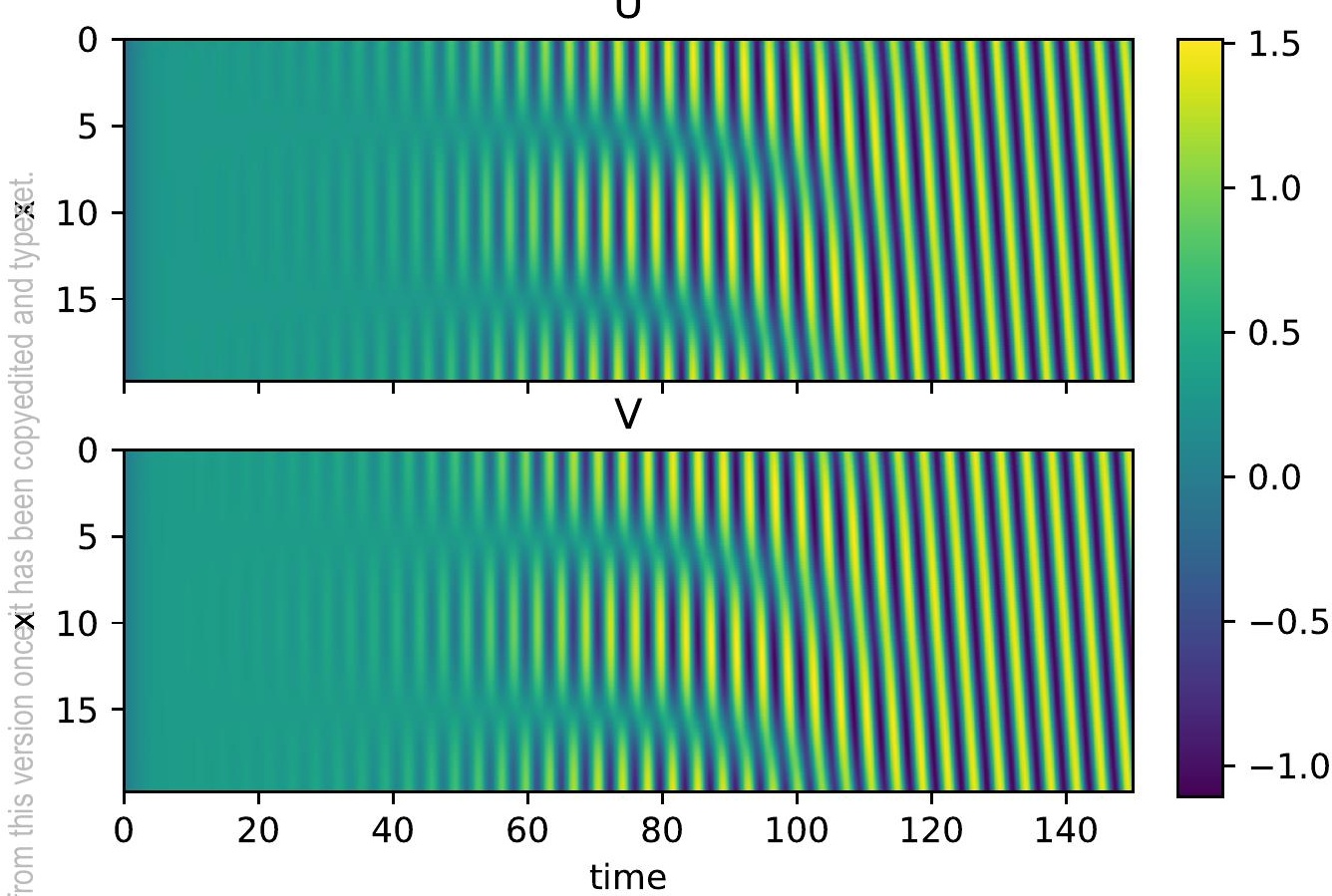




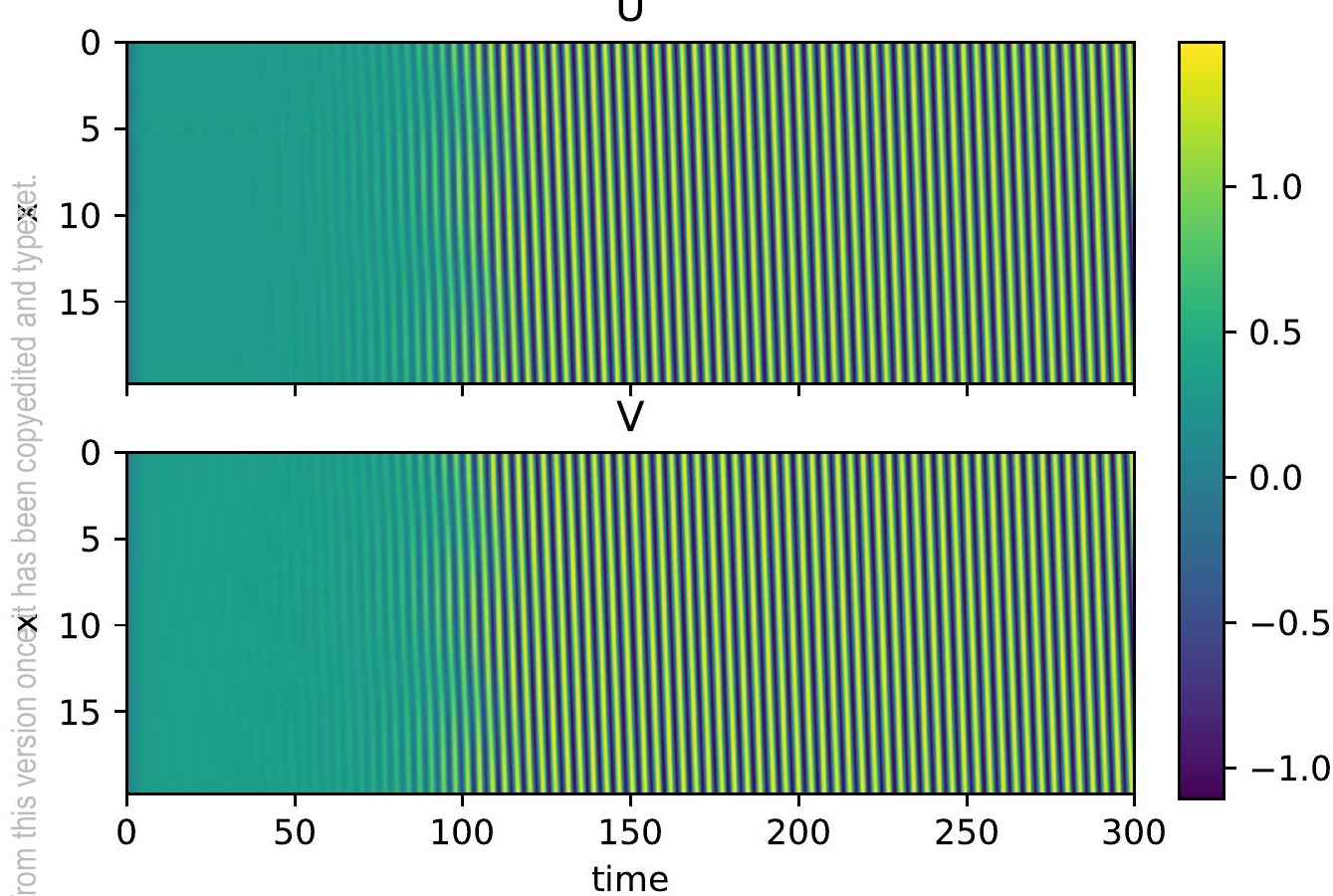

등

衰

응

일

등 흥

क्ष

(1)

핑

땋

뜽

히ㄹㅗㅗ

오 쁨

흔

늠

런

름ㅁ

응 


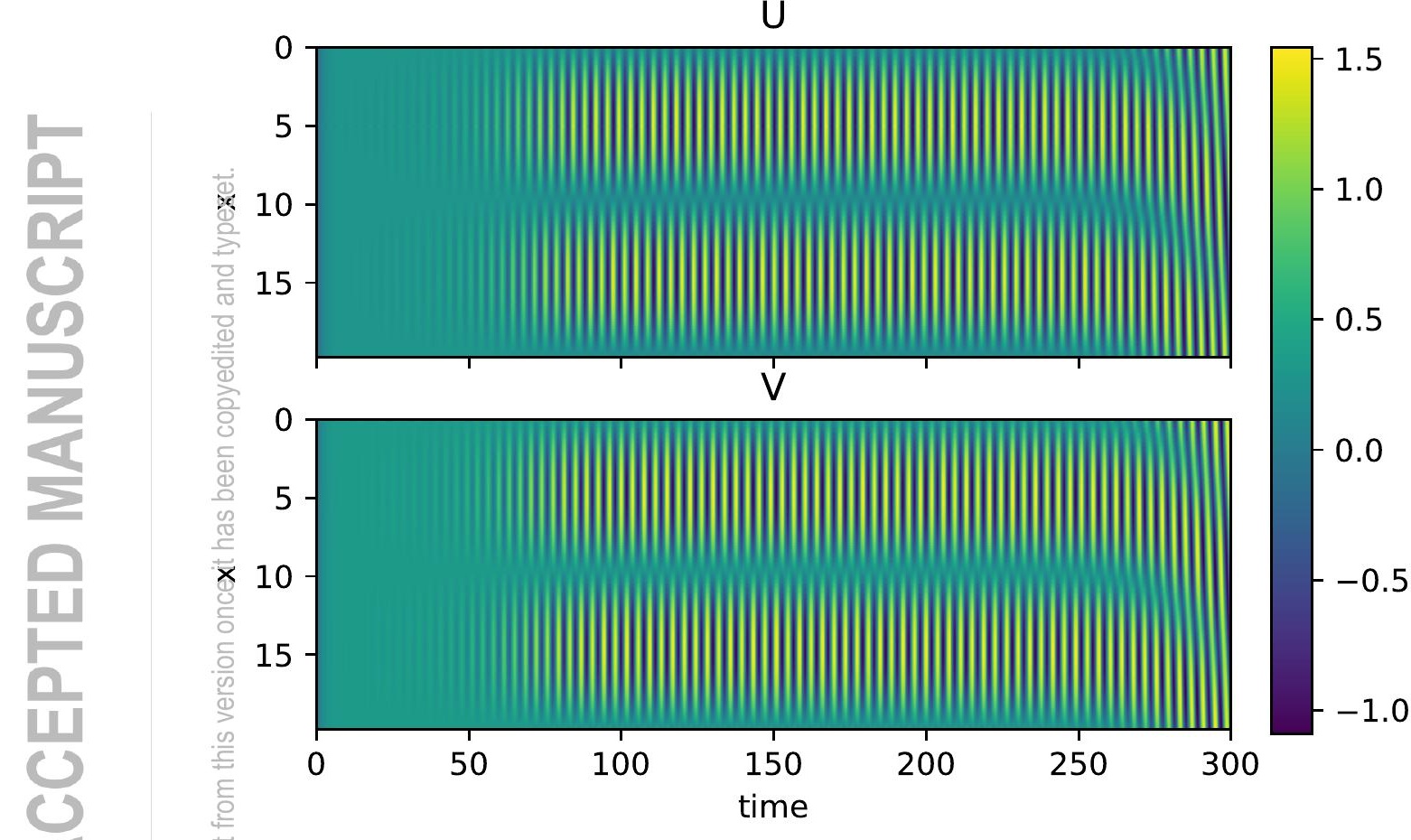




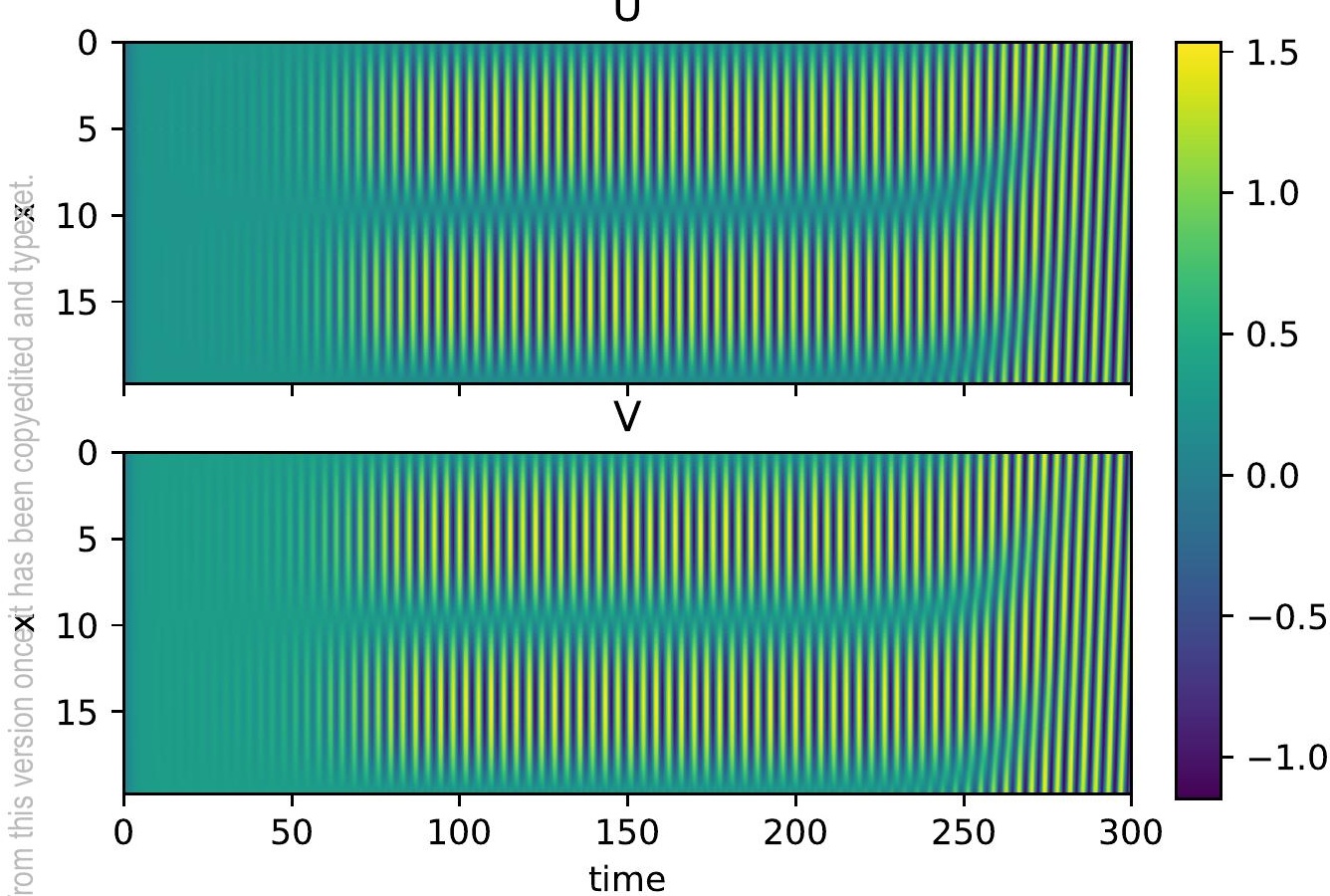

훙

응

잉

능 은

등 응

닝

(1)

등

들 만

히롬

우 뜬

흥

는

늘

है 